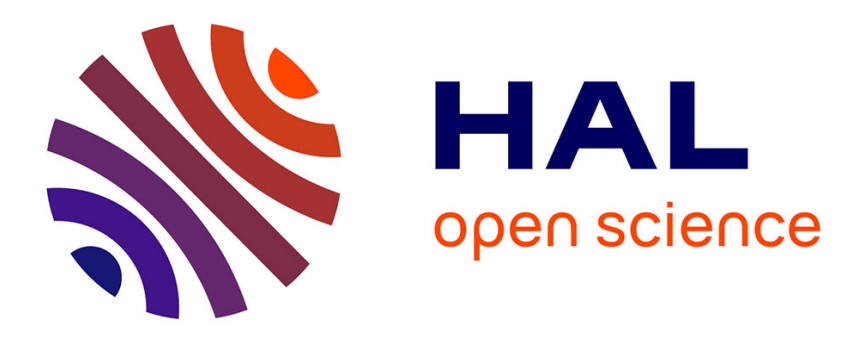

\title{
Les biens fonciers des élites sénatoriales à Constantinople et dans ses environs (451-641) \\ Vincent Puech
}

\section{To cite this version:}

Vincent Puech. Les biens fonciers des élites sénatoriales à Constantinople et dans ses environs (451641). Archimède: archéologie et histoire ancienne, 2015, 2, pp.170-193. halshs-01588083

\section{HAL Id: halshs-01588083 \\ https://shs.hal.science/halshs-01588083}

Submitted on 15 Sep 2017

HAL is a multi-disciplinary open access archive for the deposit and dissemination of scientific research documents, whether they are published or not. The documents may come from teaching and research institutions in France or abroad, or from public or private research centers.
L'archive ouverte pluridisciplinaire HAL, est destinée au dépôt et à la diffusion de documents scientifiques de niveau recherche, publiés ou non, émanant des établissements d'enseignement et de recherche français ou étrangers, des laboratoires publics ou privés. 


\section{ARCHIMĖDE N $N^{\circ} 2$}

\section{DOSSIER THÉMATIQUE : ARCHIVES DE L'ARCHÉOLOGIE}

1 Marie STAHL

Introduction : la mémoire retrouvée des archéologues

9 Marie STAHL, Lucile SCHIRR

Les archives de I'archéologie : définition, législation, état des lieux

20 Dominique BEYER, Marie STAHL (collab. Catherine DUVETTE, Isabelle WEYGAND, Françoise LAROCHE-TRAUNECKER, Marie-José MORANT, Philippe QUENET) Les archives de la composante d'archéologie orientale de I'UMR 7044

41 Cassandre HARTENSTEIN

Le fonds Montet et la statue «maussade » de Ramsès II au Palais universitaire de Strasbourg

51 Soline MORINIÈRE

Les archives de I'archéologie au SRA Alsace : état des lieux et des fonds

59 Anne ROHFRITSCH

Les archives dites « manuscrites » des membres de l'École française d'Athènes :

l'exemple des «Strasbourgeois 》 (1846-1960)

66 Cécile COURTAUD, Isabelle LESUEUR, Soline MORINIÈRE, Juliette RÉMY, Bernadette SCHNITZLER, Marie STAHL, Georges TRIANTAFILLIDIS Un projet collectif de recherche autour du fonds Arthur Stieber

78 Soline MORINIÈRE

La gypsothèque de I'Université de Strasbourg : quand les statues parlent d'elles-mêmes

\section{LA CHRONIQUE D'ARCHIMÈDE}

94 Frédéric COLIN (éd.)

La Chronique d'Archimède. Bilan des activités scientifiques 2014-2015 de I'unité mixte de recherche 7044

\section{VARIA}

134 Sarah DERMECH

Couleurs, éclat et brillance des crânes surmodelés : le cas du Néolithique Proche-oriental

150 Cinzia BEARZOT

La violence de l'État. La condamnation à mort sans jugement dans la Grèce ancienne

160 Doris MEYER

Jusqu'au dernier mot. Martyr, débat public et résistance dans la littérature de l'Antiquité tardive et à Byzance

\section{Vincent PUECH} Les biens fonciers des élites sénatoriales à Constantinople et dans ses environs (451-641)

194 Clara MILLOT

Entre les enfants d'Hérodote et les enfants d'Adam Smith. Pour une approche économique des données archéologiques

Retrouvez tous les articles de la revue ARCHIMĖDE sur :

http://archimede.unistra.fr/revue-archimede/archimede-2-2015 


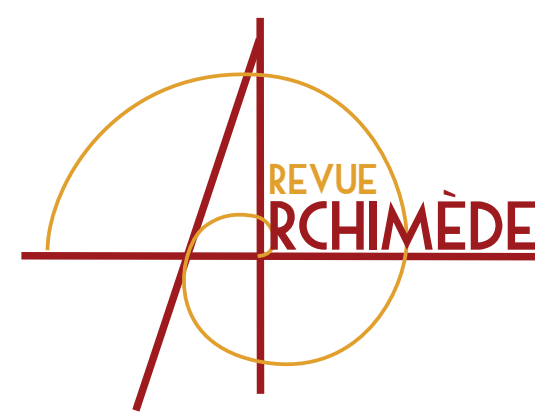

\title{
LES BIENS FONCIERS DES ÉLITES SÉNATORIALES À CONSTANTINOPLE ET DANS SES ENVIRONS (451-641)
}

\author{
Vincent PUECH \\ Maître de conférences en Histoire ancienne \\ Université de Versailles Saint-Quentin-en-Yvelines \\ vincent.puech@uvsq.fr
}

\begin{abstract}
Je suis très reconnaissant à Catherine Saliou de m'avoir invité à présenter ce travail à son séminaire de l'EPHE et de m'avoir fait maintes précieuses suggestions, en particulier pour les traductions. Je sais gré à Claire Fauchon d'avoir traduit pour moi du syriaque un passage de I'Histoire ecclésiastique de Jean d'Éphèse. Je remercie I'UMR 8167 et en particulier Emmanuelle Capet de m'avoir fourni les fonds de cartes réalisés par Fabien Tessier : ma gratitude va ainsi à Cécile Morrisson pour la carte de la ville de Constantinople (MorRIsson 2004, p. 185) et à Jean-Claude Cheynet pour la carte des environs (CHEYNET 2003, p. 317) ; la représentation cartographique des biens sénatoriaux relève bien sûr de ma seule responsabilité.
\end{abstract}

RÉSUMÉ

Les biens fonciers des élites sénatoriales à Constantinople et dans ses environs à la fin de la période antique ne sont connus que par bribes et presque seulement par les sources textuelles. Parmi les biens de 62 personnages, 45 peuvent être plus ou moins localisés, même si leur composition précise nous échappe à peu près complètement. Les biens de la famille impériale créent, à la fois en ville et dans les faubourgs, une véritable polarisation de l'espace, qui oriente la localisation de I'ensemble des propriétés sénatoriales. Nous connaissons surtout les biens de l'élite de la cour composée des patrices, consuls, préfets du prétoire, préfets de Constantinople et maîtres des milices. On peut mettre en évidence le modèle de l'oikos urbain aristocratique, centré sur la demeure du maître et incluant différents

bâtiments susceptibles de lui procurer des revenus (ate-

Mots-cLÉs

Antiquité tardive, Constantinople, sénateurs, empereur byzantin, prosopographie, biens fonciers, oikos, proasteion, Patria. liers, boutiques, bains, habitations) ou de contribuer à son prestige (églises, monastères, hospices). Il est enfin possible de déceler le rôle de l'empereur comme redistributeur des fortunes sénatoriales.
The real estates of the senatorial elites at Constantinople and in its vicinity during the end of Antiquity are fragmentary and almost only by textual sources documented. Among the estates of 62 names, 45 can be more or less localized, even if their precise composition is almost totally unknown. The estates of the imperial family create, inside as well as outside the town, a real spatial polarization, which guides the localization of the whole senatorial estates. We know above all the estates of the court elite, which includes the patricii, consuls, praefecti praetorio, prefects of Constantinople and magistri militum. One can make obviousness the model of the aristocratic urban oikos, centered on the master's habitation and including various buildings liable to get incomes for him (workshops, stores, baths, habitations) or to contribute to his prestige (churches, monasteries, almshouses). It's finally possible to discover how the emperor redistributes the senatorial fortunes.
KeYwords Late Antiquity, Constantinople, senators, byzantine emperor, prosopography, real estates, oikos, proasteion, Patria. 
« Le développement de la fortune foncière des sénateurs est un élément fondamental pour comprendre l'évolution de la classe sénatoriale elle-même » [1] : ainsi s'exprime Gilbert Dagron dans son ouvrage magistral Naissance d'une capitale. Constantinople et ses institutions de 330 à 451. Dans l'Antiquité tardive, les biens des sénateurs dans les campagnes ne sont connus que par quelques mentions fugaces dans les textes littéraires et les inscriptions. Le meilleur sort est réservé aux grandes propriétés égyptiennes relativement mieux documentées par la papyrologie [2]. Dans ce contexte d'une difficile appréhension de l'assise foncière des élites sénatoriales, les biens présents à Constantinople et dans ses environs sont davantage décelables. Non que notre connaissance en soit très complète ni très précise, mais nous pouvons approcher l'identité de certains de leurs détenteurs et, dans la plupart des cas, leur localisation, au moins approximative [3]. Dans I'ouvrage cité, G. Dagron a étudié le premier siècle de la nouvelle capitale, où les biens sénatoriaux commencent à être précisément connus à l'époque théodosienne [4].

Je voudrais ici m'intéresser aux biens fonciers des élites sénatoriales dans la capitale et ses abords à la

[1] DagRon 1974, p. 177.

[2] Voir en particulier HiCkeY 2007.

[3] Cette connaissance repose presque exclusivement sur les textes, en grande majorité littéraires et, dans une bien moindre mesure, épigraphiques. Pour la période que nous considérons, l'archéologie permet seulement de documenter les églises Saint-Jean-Baptiste de Stoudios et Saint-Polyeucte, fondées sur des biens sénatoriaux dont nous ignorons la composition. Précisément, nous nous intéresserons aux églises et monastères seulement quand ils ont été créés sur des biens sénatoriaux ; nous négligerons les fondations religieuses dont I'assise foncière est inconnue. Enfin, si nous incluons les biens des membres des familles impériales, nous ne considérons pas ici ceux des empereurs eux-mêmes, qui nous entraîneraient trop loin.

[4] Relevons ce qu'il indique à propos des propriétés suburbaines de ces élites : « les textes montrent comment toute la région de Constantinople se couvre de proasteia qui sont des période suivante, celle qui va du concile de Chalcédoine en 451 à la mort d'Héraclius en 641. Cette époque se caractérise comme celle de la fin de la civilisation antique, antérieure à la conquête arabo-musulmane, et elle bénéficie d'une relative abondance de sources. On a pu ainsi recenser les biens de 62 personnages, en éliminant ceux dont l'identification ou la chronologie étaient trop incertaines; sur ces 62 individus, les biens de 45 d'entre eux, soit plus des $2 / 3$, peuvent être localisés avec plus ou moins de précision [5]. Un tableau de la fortune des sénateurs de Constantinople au vi ${ }^{\mathrm{e}}$ siècle avait été dressé par Alexandra Čekalova [6], mais il demande à être amendé et complété, particulièrement en ce qui concerne les biens présents dans la capitale et ses environs. Parmi ces 62 personnages, les biens de 26 d'entre eux, soit pratiquement la moitié, sont pour l'essentiel connus par les Patria, des textes des VIII ${ }^{\mathrm{e}}-\mathrm{X}^{\mathrm{e}}$ siècles sur les origines de Constantinople. On sait que ces textes souvent fantaisistes véhiculent tout un imaginaire sur les débuts de la civilisation byzantine [7]. Ils doivent être sévèrement critiqués en prenant en compte leur fonctionnement interne, par exemple quand ils font de Constantin un personnage presque mythique, entouré

domaines sénatoriaux : domaine de Promotus sur lequel vivent des moines goths, domaine d'Eleutheros où le moine Hypatios s'installe à proximité de la capitale, domaine de Saturninus où Isaac et Dalmatios fondent leur monastère, domaine de Rufin - du côté de Chalcédoine - où est situé le monastère de Roufinianai, domaine de Gélanios sur lequel est édifié la colonne de Daniel, domaine de Stoudios, domaine d'Eusébia acheté par le consul Flavius Caesarius, et tant d'autres dont les dimensions ne sont peut-être pas très importantes, mais qui ont laissé une marque dans la toponymie de Constantinople et des régions environnantes » (DAGRON 1974, p. 185).

[5] Toutes les références érudites sont présentes dans l'annexe prosopographique, où les personnages sont classés par ordre alphabétique. En ce qui concerne les données échappant aux biens fonciers, nous renvoyons aux notices de la $P L R E$, dont nous discutons cependant parfois le contenu.

[6] ČEkalova 1998, p. 127-129.

[7] DAGRON 1984. 
de sénateurs dont les noms peuvent renvoyer à des individus postérieurs à lui. Néanmoins, dans le corpus du $x^{e}$ siècle, les mentions des biens fonciers sénatoriaux, qui ont souvent donné naissance à des quartiers médiévaux, inspirent largement confiance [8]. Elles sont en effet souvent rapportées aux règnes des empereurs et, dans presque tous les cas, des sources antiques corroborent l'existence des personnages à ce moment-là [9]. Il est vrai que les Patria insistent sur les personnages les plus en vue car sur 26 individus mentionnés par cette source, on recense 7 membres des familles impériales et 11 patrices. Par ailleurs, les Patria évoquent souvent les églises ou monastères installés sur les biens sénatoriaux, soit originellement par leurs détenteurs, soit plus tard. Or ces établissements religieux sont en général bien connus par de riches sources médiévales, ce qui contribue beaucoup à leur localisation. Raymond Janin avait ainsi dressé les premiers répertoires topographiques de Constantinople [10], qui conservent leur utilité. Mais la localisation des biens connus par les Patria a été entièrement revue par Albrecht Berger [11], dans un travail que j'ai abondamment utilisé.

Avant d'en venir à la période qui va du milieu du ve au milieu du $\mathrm{VII}^{\mathrm{e}}$ siècle, rappelons la composition de la fortune sénatoriale constantinopolitaine la mieux connue, celle de la fameuse amie de Jean Chrysostome Olympias, à la fin du IV siècle. Olympias [12] est la petite-fille du préfet du prétoire Ablabius (sous Constantin), la fille de Seleucus (comte honoraire) et l'épouse du comes rei privatae puis préfet de Constantinople Nebridius (mort en 386) [13]. Devenue veuve et diaconesse, elle donne ses biens à l'Église de Constantinople : 10000 livres d'or, 100000 livres d'argent, des domaines dans les provinces de Thrace, Galatie, Cappadoce première et Bithynie et des biens situés à Constantinople et dans ses environs.

[8] On peut citer ce qu'en dit Magdalino 1996, p. $16: \ll(\ldots)$ tout en reconnaissant ce qu'il y a d'illusion et de trompeI'œil dans la culture des recueils du $x^{e}$ siècle, on peut se demander si celle-ci fausse tout à fait la réalité. Je crois que non (...) ».

[9] Seuls trois personnages mentionnés par les Patria ne sont connus par aucune autre source : le patrice Bassus, le patrice et questeur du palais Claudius et le cubicularius Platon. Il faut préciser que si le premier est attribué au règne de Justinien, les deux autres sont évoqués à propos de celui de Basiliscus (475-476) ; or ce personnage qui disputa le pouvoir à Zénon est considéré par la tradition comme un usurpateur, monophysite de surcroît, ce qui explique largement les silences des sources au sujet de son règne.

[10] JANin 1964 ; JANIN 1969 ; JANIN 1975.

[11] Berger 1988.

[12] PLRE I : Olympias 2. Sur le personnage, voir DeSTEPHEN 2012.
Les propriétés présentes dans la capitale et ses abords sont les seules qui soient connues avec quelque détail. Citons la Vie d'Olympias rédigée par un auteur anonyme qui a personnellement connu cette dame : «(...) les immeubles qu'elle possédait dans la capitale, celui qui, proche de la très sainte Grande-Église, s'appelait maison d'Olympias, avec les bâtiments du tribunal, des thermes parfaitement aménagés et tous les édifices situés à côté, ainsi que le Silignarion ; puis, près des thermes publics de Constance, la maison qui lui appartenait, et dans laquelle elle demeurait, et enfin cette autre maison à elle qu'on appelait « maison d'Évandre », ainsi que toutes ses propriétés des faubourgs »[14]. Selon la reconstitution d'Anne-Marie Malingrey, les biens d'Olympias semblent ainsi se répartir en trois groupes : un premier aux abords de Sainte-Sophie comprenant des thermes et une boulangerie (silignarion), un deuxième proche des thermes de Constance et donc des Saints-Apôtres et un troisième hors les murs incluant la maison dite d'Évandre et divers proasteia. Olympias, une fois devenue diaconesse, construisit un monastère attenant à Sainte-Sophie, donc présent dans le premier secteur évoqué [15]. Ce monastère passa en succession à ses parentes, Marina et Elisanthia [16].

\section{LA TERMINOLOGIE}

Les éléments de la fortune d'Olympias posent déjà presque tous les problèmes de la composition des biens sénatoriaux entre 451 et 641 , et en particulier celui du vocabulaire employé pour les désigner. Les termes utilisés sont principalement au nombre de quatre, que je cite par ordre décroissant du nombre de mentions: oikos, oikia (ou domus), palation (ou palatia) et proasteion [17]. Les trois derniers ne présentent guère de difficulté d'interprétation.

[13] Delmaire 1989b, p. 88-92 : éduquée par la sœur de l'évêque d'Iconium, Amphiloque, Olympias paraît donc bien issue d'une famille du centre de I'Asie Mineure, ce que confirme la localisation de ses biens.

[14] Vie d'Olympias, V, I. 25-33, p. 416-418: $\tau \grave{\alpha} \varsigma$

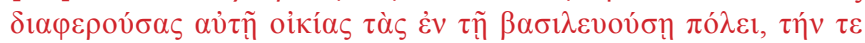

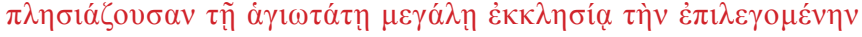

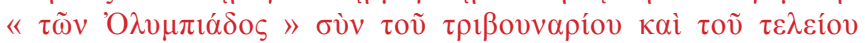

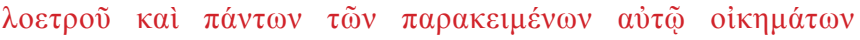

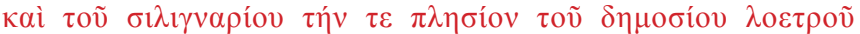

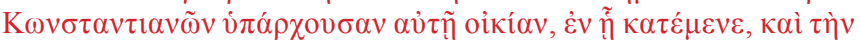

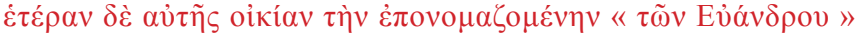

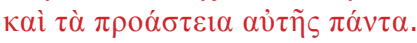

[15] Vie d'Olympias, VI, p. 418.

[16] Vie d'Olympias, XII, p. 432.

[17] On trouve néanmoins aussi ousia (Eulogius), praitôrion (Hierius) et aulè (Justinien), que I'on peut traduire dans leur contexte respectivement par «propriété », «palais » et « résidence ». 
L'oikia ou domus désigne en général la maison urbaine : le terme est cité 10 fois [18] et peut être suspecté dans 6 cas [19]. Le terme de palation, plus souvent remplacé par son pluriel palatia, désigne le palais, soit une demeure dont I'ampleur mérite d'être signalée : il est employé 6 fois, toujours à propos de membres de la famille impériale [20]. Un proasteion désigne un domaine suburbain ou un faubourg entier [21] : nous disposons d'un faible nombre d'attestations du terme [22], mais il était évidemment plus répandu et peut d'ailleurs souvent être soupçonné grâce à la localisation ou à la description du bien [23].

C'est le terme d'oikos, le plus répandu, qui pose davantage de problèmes. On sait qu'en grec classique il peut désigner à la fois la maison et l'ensemble des biens. Pour le monde byzantin, le terme est encore plus polysémique, mais, si l'on se limite à notre propos, on peut en citer quelques définitions proposées par les historiens. Pour G. Dagron, « la réussite d'un individu suppose, pendant toute cette époque, sa venue à Constantinople, son enrichissement, et l'achat, grâce à cette fortune nouvelle, d'un terrain (à bâtir ou déjà bâti) dans la capitale, un oĩkos qui soit à la fois son oikía (une résidence lui donnant une « surface ») et un capital investi »[24]. Selon Michel Kaplan, « le modèle de l'oikos, c'est une administration centralisée en un point, là où réside le propriétaire des biens et où doivent donc converger les revenus tirés de ceux-ci »[25]. Il en ressort donc que l'oikos urbain aristocratique est centré sur la demeure du maître et inclut différents bâtiments susceptibles de lui procurer des revenus (ateliers, boutiques, bains, habitations) ou de contribuer à son prestige (églises, monastères, hospices) [26]. Nous retrouvons ainsi la fortune d'Olympias. À son sujet, le terme employé pour désigner les maisons urbaines est oikia.

[18] Oikia est employé à propos d'Andreas, Apion, Dagalaiphus, Germanus, Jean de Cappadoce, Marinus, Maxentianus, Pierre Barsymès et Philippicus ; domus est cité au sujet de Germanus et Maxentianus par les Acta Conciliorum Ecumenicorum, de Marinus et Pompeius par Marcellinus comes.

[19] D'après le texte syriaque de Jean d'Éphèse, il s'agit de Pierre Barsymès, du frère de l'empereur Justin II, Marcellus, et de membres de la famille de l'empereur Maurice: son père Paulus (pour 2 maisons), son frère Petrus, sa sœur Gordia et son beau-frère Philippicus, sa sœur Theoctista. Le terme employé est beth, usuel en syriaque pour désigner la maison.

[20] Ce sont les cas d'Anastasia, Justinien, Philippicus, Probus, Sophia et Vigilantia.

[21] Étienne 2013.

[22] On le trouve au sujet d'Ardabur, Bélisaire, Hierius et Nicetas. Pour Gelanius, le terme employé est celui d'agros (champ), ce qui renvoie évidemment à un proasteion.

[23] Dans les cas de Dexicrates, Marcellus, Paulus, Pierre le Patrice, Petrus et d'Urbicius (préposite). Marcellus, Paulus et
Mais I'ensemble, en particulier le groupe de biens proche de Sainte-Sophie, a tous les caractères d'un oikos, puisqu'il comprend des habitations, des thermes, une boulangerie, un monastère et qu'il assure la gestion de domaines suburbains. Retenons encore la formule de G. Dagron à propos d'Olympias : « nous voyons par quel biais I'oikia, ensemble immobilier, peut devenir l'oikos byzantin, une fondation gérant un capital (généralement foncier) dont les revenus sont affectés à une certaine utilisation »[27].

Pour notre période, le cas le plus précis d'oikos que nous connaissions se compose des biens légués par le patrice et préfet du prétoire d'Orient (sous Anastase) Hierius à son fils Constantinus et au fils de ce dernier le clarissimus Hierius. Ces biens font partie du proasteion de ta Kôparia, qui comporte en effet tous les caractères d'un oikos. Il comprend des palais, des ports, des locaux et ateliers, des bains, des jardins, un hippodrome et une citerne. Notons qu'un hippodrome privé est également connu dans le cas de l'oikos du maître des milices et comes excubitorum Priscus (sous Maurice). Alors que I'hippodrome appartenant à Hierius a toute chance de se trouver à l'extérieur de Constantinople, puisqu'il fait partie d'un proasteion, celui de Priscus est intégré dans le quartier urbain de ta Boraidou, au nord du port Sophien. De tels hippodromes intégrés dans les palais aristocratiques sont aussi connus dans la Rome du début du ve siècle [28]. Il apparaît que les sénateurs imitaient ainsi les palais impériaux, qui comprenaient à l'occasion de tels hippodromes, tel celui de Domitien sur le Palatin [29] ou de Maxence sur la Via Appia [30].

Si I'on revient à l'ensemble des données sur les biens sénatoriaux entre 451 et 641 , on constate que le terme d'oikos est employé 22 fois. La très grande majorité de ces usages, 18 exactement, est le fait des Patria [31].

Petrus sont cités par Jean d'Éphèse, qui emploie un terme syriaque directement issu du grec proasteion.

[24] DAGRON 1977, p. 8.

[25] KAPLAN 1992, p. 148.

[26] Pour la période médiévale, voir MAGDALINo 1974.

[27] DAGRON 1974, p. 505-506.

[28] Olympiodore, fr. 41, p. 204 : « chacune des grandes mai-

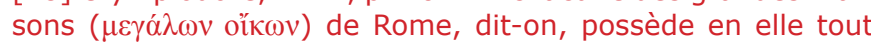
ce qu'une cité de bonne taille doit posséder : un hippodrome, des places, des temples, des fontaines et des bains différenciés » (trad Dubouloz 2011, p. 279 n. 42). Dubouloz 2011, p. 279 , signale aussi que la villa des Tusci appartenant à Pline le Jeune ( $\mathrm{II}^{\mathrm{e}}$ siècle) comportait un hippodrome.

[29] Gros 2006, p. 258.

[30] Donciu 2012, p. 137-138 (il existe cependant un débat sur l'intégration du cirque au palais suburbain).

[31] À propos d'Amantius, Anthemius, Areobindus, Armatus, Aspar, Basilides, Basiliscus, Bassus, Bonus, Claudius, Euphémie, Illous, Narsès, Pierre le Patrice, Platon, Smaragdus et Urbicius. 
Oikos est utilisé 4 fois par des sources anciennes (remontant aux $\mathrm{VI}^{\mathrm{e}}$, $\mathrm{VII}^{\mathrm{e}}$ et $\mathrm{VIII} / \mathrm{e} \mathrm{IX}^{\mathrm{e}}$ siècles) : Malalas, la Vie de Théodore de Sykéôn, Jean d'Antioche et Théophane, à propos respectivement de Marinus, Domnitziolus, Priscus et Eugenius [32]. Or la présence du terme dans les Patria pourrait être liée à une perception des biens aristocratiques au $x^{e}$ siècle, sans qu'elle reflète fidèlement la situation antique. Paul Magdalino a en effet montré que la structure de l'oikos s'était généralisée au $x^{e}$ siècle, mais, en même temps, il insiste sur la continuité du phénomène depuis la fin de l'Antiquité [33]. Nous verrons que dans 11 cas mentionnés par les Patria, soit la moitié des mentions totales, le terme d'oikos s'accompagne de constructions (bains, citernes, églises, monastères, hospices) qui lui donnent une réalité concrète [34]. Par ailleurs, on l'a vu, quelques auteurs de la période ou un peu postérieurs à celle-ci, utilisent aussi le terme, sans que nous connaissions, dans leur cas, de bâtiments associés. Au total, il est donc probable que le terme d'oikos est employé largement à bon escient dans les différentes sources, sans que I'on puisse exclure que, dans quelques occurrences, il soit un synonyme d'oikia ; il est également possible que les termes d'oikia ou domus puissent désigner dans certains cas un oikos, ainsi que nous l'avons vu à propos d'Olympias [35]. À la suite de G. Dagron, on peut renvoyer enfin aux données procurées par la Notitia urbis constantinopolitanae, un texte remontant au règne de Théodose II, soit juste avant la période que nous considérons. Ce texte compte 5 palatia, 6 domus divinae Augustarum et 3 domus nobilissimae, toutes catégories appartenant à des princesses de la dynastie théodosienne [36]. Par ailleurs, il affirme que Constantinople renferme 4388 domus, qui semblent bien regrouper tous les types d'habitations, équivalant, pour Rome, à la fois aux domus aristocratiques et aux insulae réservées au peuple [37]. Les biens aristocratiques autres que ceux de la famille impériale ne sont donc pas nettement caractérisés dans la Notitia urbis constantinopolitanae, et c'est ce qui fait tout l'intérêt d'une étude de la période postérieure.

[32] L'existence d'un oikos peut être soupçonnée dans les cas, connus par Jean d'Éphèse, de Marcellus, Pierre Barsymès, Paulus, Petrus, Philippicus et Theoctista.

[33] Magdalino 1996, p. 48 : «Constantinople est restée, au $x^{e}$ siècle, la ville chrétienne antique qui avait pris forme au cours des deux siècles et demi suivant sa fondation » (la première partie de l'ouvrage s'intitule de façon significative «La survie de la Mégalopolis paléochrétienne »).

[34] Au sujet des biens d'Amantius, Anthemius, Bonus, Illous, Narsès, Olybrius, Pierre le Patrice, Philippicus, Platon et Urbicius. Des églises sont aussi associées à deux palatia, ceux de Probus et Vigilantia, et aucun terme précis n'accompagne l'église fondée par Sporacius ni le monastère créé par Studius.

\section{LA FAMILLE IMPÉRIALE}

Comme à l'époque théodosienne, la famille impériale est la première concernée par la détention de palais urbains [38]. Anthemius, gendre de Marcien et empereur d'Occident, détenait un oikos entre les murailles de Constantin et Théodose, à l'ouest de la citerne de Saint-Mokios. Il disposait aussi de biens sur la rive asiatique du Bosphore. Olybrius, gendre de l'empereur $d^{\prime}$ Occident Valentinien III et consul en Orient en 464 (avant d'être lui-même empereur d'Occident en 472), détenait un oikos aux Constantinianae. La sœur de Léon

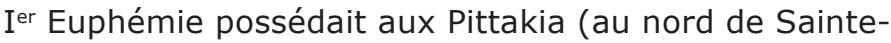
Irène) un oikos, près duquel elle fit ériger une statue en I'honneur de son frère. Basiliscus, le frère de l'impératrice Vérine (épouse de Léon I ${ }^{\mathrm{er}}$ ) détenait au nordouest du forum de Constantin un oikos qu'il fit transformer en palais quand il usurpa le trône sous le règne de Zénon, en 475-476. Le neveu de Vérine et Basiliscus, Armatus, disposait d'un oikos au nord-est des SaintsApôtres. Le neveu d'Anastase, Probus, était certainement le patrice Probus qui possédait au nord du port Sophien un magnifique palais. Cette demeure fit sans doute partie des biens du personnage confisqués par Justinien à la suite de la révolte Nika de 532 mais qui lui furent restitués dès 533. On sait en outre qu'en 542 Probus logea Jean d'Éphèse à Constantinople, probablement dans le même palais. Un autre neveu d'Anastase et cousin du précédent, Pompeius, possédait avec certitude une maison à Constantinople : on sait qu'elle fut incendiée lors des émeutes de 512. Le futur Justinien $\mathrm{I}^{\text {er }}$ résidait avant son avènement au palais d'Hormisdas qui se trouvait immédiatement à l'est du port Sophien. Le cousin de Justinien Boraides possédait des biens en arrière du port Sophien. Le même personnage disposait d'un domaine (proasteion) vers le centre du Bosphore, sur sa rive asiatique, à peu près en face de Sôsthenion. On comprend que l'héritage de Boraides ait été disputé : on sait qu'il légua l'essentiel de ses biens à son frère

[35] Dans le cas de Marinus, Marcellinus comes emploie domus et Malalas oikos, ce qui laisse penser que ce vocabulaire peut être fluctuant. Dubouloz 2011 montre qu'à Rome une domus a très souvent les caractères de l'oikos de Constantinople.

[36] DAGRON 1974, p. 97.

[37] DaGron 1974, p. 525-527. Dubouloz 2011, p. 568 n. 102, pense que ces 4388 domus « désignent toutes les propriétés sénatoriales ». Il semble que ce serait un chiffre trop important, qui doit donc plutôt renvoyer à l'ensemble des domus et insulae. Mais dans ce cas domus paraît être employé plutôt au sens d'oikos que d'oikia.

[38] Voir la carte 1. J'insiste sur les lacunes des sources et le caractère souvent approximatif des localisations, qui rendent cette carte et la suivante nécessairement incomplètes et imprécises. 
Germanus et aux fils de ce dernier, mais que Justinien intervint pour que la fille de Boraides reçoive davantage que le minimum légal. Germanus, cet autre cousin de Justinien, détenait certainement une maison proche du port Césarien, donc à proximité des biens de Boraides, vers le sud-ouest. Vigilantia, sœur de Justinien $\mathrm{I}^{\mathrm{er}}$ et mère de Justin II, reçut de son frère l'empereur un palais situé au nord du forum de Théodose. La nièce de Théodora, Sophie, I'épouse de Justin II, reçut dès le règne de Justinien, le palais dit Sophien : il était vraisemblablement situé entre les forums de Constantin et Théodose. Sous le règne de Tibère II, Sophie fut confinée dans ce palais après un soupçon de complot contre l'empereur. Marcellus, frère de Justin II, disposait de maisons et de biens fonciers impossibles à localiser. Anastasie, femme de Tibère II et belle-mère de Maurice, reçut de ce dernier le palais Sophien. Maurice partagea entre son père Paulus et son frère Petrus les maisons et biens fonciers qui appartenaient auparavant au frère de Justin II, Marcellus. Maurice donna aussi à son père et à sa mère une maison proche de Sainte-Sophie et du Grand Palais. Le même empereur attribua à sa sœur Theoctista I'ancienne maison de Pierre Barsymès, ministre de Justinien. Enfin, il céda à sa sœur Gordia et à son époux Philippicus une vaste demeure dans le quartier du Zeugma ; on ne sait si ce bien est identique à la maison que fit bâtir Philippicus à Constantinople, aux dires de Théophane. En outre, le même Philippicus fit construire à Chrysopolis un palais destiné à recevoir l'empereur Maurice et ses enfants. Sous le règne de Phocas, son gendre et ancien protecteur Priscus disposait d'un oikos dans le quartier de ta Boraidou, en arrière du port Sophien. Le neveu de Phocas Domnitziolus possédait un oikos aux Arcadianae, au nord-est de Sainte-Sophie.

Si I'on dresse le bilan des biens des familles impériales, on constate une concentration dans trois zones. Au nord du Grand Palais, dans les environs de SainteSophie, sont concernés Euphémie (sous Léon $\mathrm{I}^{\mathrm{er}}$ ), Paulus (sous Maurice) et Domnitziolus (sous Phocas). Dans le secteur du port Sophien sont surtout concentrés les biens de Justinien et de ses cousins Germanus et Boraides, mais également ceux de Probus (sous Anastase) et Priscus (sous Phocas). Le troisième secteur se situe entre les forums de Constantin et Théodose, avec les biens de Basiliscus, Vigilantia (sous Justinien) et le palais de Sophie, épouse de Justin II, passé à Anastasia, épouse de Tibère II. Quelques membres des familles impériales sont aussi présents dans toute la zone qui va de la Mésè jusqu'à la Corne d'Or, avec Olybrius (sous Léon I ${ }^{\mathrm{er}}$ ), Armatus (sous Zénon) et Philippicus (sous Maurice).

\section{LA HIÉRARCHIE SÉNATORIALE}

En dehors de la famille impériale, nous connaissons surtout les biens des patrices, au nombre de 25 . Sous Marcien, c'est le cas des maîtres des milices Aspar et son fils Ardabur ainsi que de Basiliscus, mais aussi de l'épouse du patrice Zénon Paulina et du consul Studius. Sous le règne de Zénon sont attestés les biens du maître des offices puis maître des milices Illous, du consul Dagalaiphus, ainsi que de Claudius, questeur du palais pendant I'usurpation de Basiliscus. Sous Anastase, nous trouvons le préfet du prétoire Hierius, les maîtres des milices Rufinus et Urbicius et le consul Dexicrates. Sous Justin $\mathrm{I}^{\mathrm{er}}$, il est peut-être question d'Eulogius. Sous Justinien, c'est le cas du consul originaire d'Égypte Apion, du curateur de maison divine Aetherius, des préfets du prétoire Basilides, Jean de Cappadoce et Pierre Barsymès (qui fut aussi comte des largesses sacrées), ainsi que du maître des milices Bélisaire et du praepositus sacri cubiculi Narses. Sous Justin II nous connaissons les biens d'Eusebius, sous Tibère II, ceux de Smaragdus et, sous Héraclius, ceux du maître des milices Bonus et du comes excubitorum Nicetas. En dessous des patrices sont attestées les propriétés de six simples consuls : le consul de 410 ou 456 Varanes, le comes domesticorum peditum Sporacius (sous Marcien), le consul de 457 ou 492 Rufus, le maître des milices Areobindus et son épouse Iuliana Anicia (sous Anastase), le curateur de maison divine Anatolius et le maître des milices Maxentianus (sous Justinien). En dehors des membres des familles impériales, des patrices et consuls, nous appréhendons les biens du praepositus sacri cubiculi Urbicius (entre les règnes de Marcien et Anastase), du castrensis Gelanius (sous Léon $\mathrm{I}^{\mathrm{er}}$ ), des cubiculaires Platon (sous Basiliscus) et Andreas (sous Justin $\mathrm{I}^{\mathrm{e}}$ ), des préfets du prétoire Marinus (sous Anastase) et Eugenius (sous Justinien), des préfets de Constantinople Ioannes Cocorobius et Musonius, du maître des milices Dagisthaeus et de I'illustris Meltiades (sous Justinien).

$\mathrm{Au}$ total sont particulièrement bien représentés les préfets du prétoire, les préfets de Constantinople et les maîtres des milices, trois fonctions qui constituent exactement le sommet de la hiérarchie administrative. Du point de vue de la localisation des biens, on note une concentration à l'extrémité de la presqu'île, dans la partie orientale de l'espace urbain enserré dans la muraille de Constantin. Il est plus original de constater que les propriétés sénatoriales se regroupent selon la même logique que les biens des familles impériales, soit dans les trois zones que constituent les environs de SainteSophie, le port Sophien et le secteur compris entre les 
forums de Constantin et Théodose. Mais elles occupent également d'autres espaces où elles ne sont jamais isolées, et se regroupent au moins par deux, ce qui, en raison des lacunes des sources, doit signifier que d'autres biens encore étaient présents. Le premier secteur est celui du Zeugma, en face de Galata, le deuxième est la vallée centrale du Lycus, de part et d'autre de la muraille de Constantin, le troisième est celui de Psamathia, au sud-ouest, entre les enceintes de Constantin et de Théodose, et le quatrième est le Cosmidion, au nordouest, au-delà de la muraille de Théodose.

Les biens des sénateurs présents dans les environs de Constantinople se regroupent en trois zones géographiques [39]. La plus proche de la ville se situe dans le secteur des Blachernes, immédiatement au nord-ouest de la muraille de Théodose. Y sont présents les biens de Paulina, Hierius (et son fils Anthemius) et Nicetas, déjà évoqués. Si I'on s'éloigne de Constantinople dans la même direction, vers le nord-ouest, on trouve les domaines de Dexicrates et Urbicius, dont une borne a été retrouvée aux alentours de Küçük Köy. La deuxième zone est la rive européenne du Bosphore, où nous ne connaissons que deux domaines, celui de Gelanius à Anaplous (Arnavutköy) et celui d'Ardabur passé à Hierius à Sôsthenion (Istinye). La majorité des propriétés suburbaines connues est présente dans la troisième zone, la rive asiatique du Bosphore et surtout celle de la mer de Marmara. Sur le détroit se trouvent les biens d'Anthemius et Boraides, à proximité de Kanlica, en face d'Istinye. Mais c'est sur la mer de Marmara que se concentre la plus grande partie des biens, avec, du nord au sud, ceux de Philippicus à Chrysopolis (Üsküdar), Rufus dans les environs de Chalcédoine (Kadiköy) sans doute à Bulgurlu, Pierre le Patrice avec la petite île d'Akonai située en face de Chalcédoine et enfin Bélisaire, qui possédait le domaine des Rufinianae (à Caddebostan) et un bien à Panteichion (Pendik). On constate que les biens des personnages les plus proches des familles impériales sont présents dans cette dernière zone de la rive asiatique de la mer de Marmara, à proximité des îles des Princes. Par ailleurs, sur 14 détenteurs de biens connus dans les environs de Constantinople, 11 sont des patrices, ce qui montre que ces domaines suburbains étaient largement monopolisés par l'élite de la cour.

La composition de ces proasteia suburbains est mal

[39] Voir la carte 2

[40] Voir Morvillez 2014 et Saliou 2014

[41] CABouret 2013 a mis en valeur I'association des modes de vie urbain et rural chez les élites.

[42] La fonction publique des oikoi privés a été mise en lumière par GAScou 1985. Pour le débat sur cette conception : CARRIÉ 1999, p. 351-352. connue, mais ils apparaissent principalement voués aux jardins et à la viticulture. Des jardins [40] sont attestés dans les cas de Hierius et Philippicus et pour ce dernier s'ajoutent des viviers pour l'élevage des poissons. Des vignes sont connues pour Bélisaire et Gelanius. Notons que, tant dans le cas des jardins que dans celui des vignes, les mentions dont nous disposons concernent les deux rives, européenne et asiatique, ce qui semble bien indiquer le caractère général de ces phénomènes. Par ailleurs, les quatre personnages mentionnés disposaient aussi de biens urbains, ce qui montre que ces proasteia associaient des fonctions de villégiature [41] et de production agricole, probablement à visée autarcique dans le cadre d'un oikos.

\section{LA VOCATION PUBLIQUE DES BIENS}

Les propriétés sénatoriales ont vu, au-delà de leur fonction d'habitations privées, l'édification de constructions à vocation publique [42]. Nous connaissons ainsi 10 églises et 2 monastères, 2 bains et 2 citernes, 3 hospices. Les mieux représentés sont donc les bâtiments religieux [43]. Les fondateurs d'églises sont sous Marcien Anthemius (Saint-Thomas) et Sporacius (Saint-Théodore), sous Léon $\mathrm{I}^{\mathrm{er}}$ Olybrius (SainteEuphémie) [44], sous Basiliscus Platon (Saint-Procope), sous Anastase Amantius (Saint-Thomas), Probus (SaintThéodore) et Urbicius (Théotokos), sous Justinien Narses (Saints-Probus-Tarachus-et-Andronicus), Pierre le Patrice (Théotokos) et Vigilantia (Saint-Procope). On note au passage une prédilection pour la Théotokos et les saints Procope, Théodore et Thomas, tous représentés deux fois. Les deux monastères sont fondés I'un en ville par le célèbre patrice Studius (dédié au Prodrome) et l'autre à Chrysopolis par le beau-frère de l'empereur Maurice, Philippicus (voué à la Théotokos). Les hospices sont tous destinés aux vieillards ( $\gamma \eta \rho \kappa о \mu \varepsilon \tilde{\alpha} \alpha)$ : ils furent créés par Anthemius, Narses et Pierre le Patrice. Enfin, des bains furent fondés par Anthemius et le patrice Smaragdus et des citernes par les patrices Illous et Bonus. Au total, les trois types de constructions furent créés par Anthemius, tandis que Narses et Pierre le Patrice fondèrent à la fois églises et hospices. Ces derniers, coûteux, relevèrent toujours de bienfaiteurs qui investirent aussi dans d'autres

[43] Sur l'abondante législation des $\mathrm{V}^{\mathrm{e}}$ et $\mathrm{VI}^{\mathrm{e}}$ siècles sur les fondations religieuses privées, voir FLusin 1998, p. 491-493.

[44] Il faut noter que Sainte-Euphémie fut commencée par la belle-mère d'Olybrius, Eudoxia, puis embellie par Olybrius et son épouse Placidia et enfin par leur fille Anicia Iuliana. Les biens de cette dernière et ceux de son époux Areobindus étaient voisins de ceux d'Olybrius. 
types de constructions. Il faut souligner que ces trois personnages, Anthemius, Narses et Pierre le Patrice, étaient très proches des empereurs Marcien et Justinien et bénéficiaient à l'évidence de fortunes tout à fait conséquentes. Plus généralement, l'agglomération dans un oikos de bâtiments de vocations différentes a été ainsi décrite par P. Magdalino à propos des bains : «bien avant de tomber en ruines, les grands thermes publics à entrée gratuite cédèrent la place, dès le $\mathrm{vI}^{\mathrm{e}}$ siècle, à des installations balnéaires plus modestes, rattachées à des fondations religieuses, où l'entrée était payante » [45]. Si cette situation paraît s'être généralisée à partir du vi ${ }^{\mathrm{e}}$ siècle, notons cependant qu'Olympias possédait déjà de tels bains à la fin du Ive siècle. En outre, les thermes privés ouverts au public moyennant un droit d'entrée sont bien connus à Antioche dans I'Antiquité tardive [46] et à Rome pendant toute la période impériale [47]. Par ailleurs, le rôle tout particulier des constructions religieuses dans la constitution des oikoi a été ainsi décrit par G. Dagron : «les Patria montrent par un très grand nombre d'exemples que I'oĩkos privé ne devient véritablement quartier que par la fondation d'un établissement charitable ou cultuel, beaucoup plus rarement d'un "palais" ou d'un bain » [48].

\section{LES CHANGEMENTS DE PROPRIÉTAIRES}

Nos sources laissent entrevoir que quelques-uns de ces biens changèrent de propriétaires. Le passage du domaine ( $\pi \rho o \alpha ́ \sigma \tau \varepsilon ı v)$ du patrice Ardabur (sous Marcien) entre les mains du clarissimus Hierius et de son fils Anthemius (sous Justinien) n'est qu'allusivement connu par la Novelle 159 [49], mais il dut relever d'un banal achat car l'acquéreur est d'un niveau social inférieur au détenteur originel. Dans le cas de Jean de Cappadoce, Marcellinus comes [50] rapporte clairement que sa maison fut donnée par Justinien à Bélisaire à I'occasion de son exil en Égypte. Nous connaissons d'autres cas de

[45] Magdalino 1996, p. 31.

[46] Saliou 2000, p. 804. Signalons que le bain ( $\pi \rho 1 \beta \alpha ́ \tau o v)$ possédé par Ardabur à Daphné, dans la banlieue d'Antioche, renvoie au personnage connu à Constantinople par la Novelle 159 (texte qui mentionne aussi l'un de ses biens à Antioche sans que I'on sache s'il s'agit de la villa où a été retrouvée I'inscription sur mosaïque évoquant le bain).

[47] Dubouloz 2011, p. 50 (juriste Papinien de la première moitié du III $^{\mathrm{e}}$ siècle), p. 81 (Frontin sous Trajan, à propos du privilège de raccordement au réseau public d'adduction), p. 168 n. 34 (inscription mentionnant la location du balneum des praedia de Iulia Felix à Pompéi), p. 272-273 (juriste Scaeuola de la seconde moitié $d u$ II $^{\mathrm{e}}$ siècle, au sujet d'un citoyen de Tibur dont les héritiers doivent ouvrir gratuitement au public leur bain privé).

[48] Dagron 1977, p. 8-9.

[49] Novelle 159 (555), p. 736. confiscation par Justinien. Le neveu d'Anastase Probus perdit ses biens après la révolte Nika mais ils lui furent rendus [51]. Le patrice Eulogius, s'il a bien existé, les perdit définitivement dans les mêmes circonstances [52]. L'ancien préfet du prétoire Eugenius vit son oikos confisqué en 560 sans que I'on sache à qui il fut donné [53]. Les autres cas connus remontent au règne de Maurice. Nous avons la chance, grâce à Jean d'Éphèse [54], de saisir la manière dont cet empereur a distribué à divers membres de sa famille des biens ayant appartenu à Pierre Barsymès, ministre de Justinien, et à Marcellus, frère de Justin II. En outre, les Patria indiquent que le même Maurice a donné à sa belle-mère Anastasie le palais de Sophie, épouse de Justin II, ce qui rappelle le destin des biens du frère de ce même empereur [55]. La maigreur de la documentation permet quand même de mettre en valeur le rôle central des empereurs dans la distribution des biens fonciers, non seulement en ce qui concerne la famille impériale, mais aussi en faveur de l'élite sénatoriale [56]. On a l'impression que les empereurs disposent d'un jeu de biens dont ils usent au gré de leurs priorités politiques.

\section{CONCLUSION}

Il faut pour conclure évoquer plus largement les relations entre l'empereur et les fortunes sénatoriales. Certains des biens détenus par des membres de la famille impériale ou même par de simples sénateurs appartiennent en fait à l'empereur, qui les distribue selon son bon vouloir. La limite peut être ainsi ténue entre un oikos sénatorial, éventuellement prélevé sur la fortune impériale [57], et un bien impérial, une domus divina, toujours géré par un membre de l'élite sénatoriale [58]. Les biens présents dans la capitale et encore davantage ceux des faubourgs concernent prioritairement I'élite de la cour, I'entourage impérial. En dehors des membres de la famille impériale, on a noté la place toute particulière des patrices, consuls,

[50] Marcellinus comes, Chronique, add. a. 544.

[51] Malalas, Chronique, XVIII, 80, p. 403.

[52] Vie de Daniel le Scétiote, 9.

[53] Théophane, Chronographie, p. 235.

[54] Jean d'Éphèse, Histoire ecclésiastique, III, 5, 18.

[55] Patria, III, 125, p. 256.

[56] Cheynet 1991, p. 210 : « Il est avéré en tout cas que les empereurs conservèrent toujours une responsabilité primordiale dans la constitution et le maintien des plus hautes fortunes de l'Empire, ce qui était un remarquable instrument de pouvoir à leur disposition ».

[57] Sur la concession des biens de la res privata, voir Delmaire 1989a, p. 622-623.

[58] Voir KAPLAN 1986 avec en annexe une «prosopographie sommaire des curateurs ». 
préfets du prétoire, préfets de Constantinople et maîtres des milices. Il est sûr que les sources s'intéressent prioritairement à cette mince élite, mais il est également logique que cette dernière soit avantagée dans l'accès aux biens fonciers proches du palais. Il est à signaler que les biens de la famille impériale créent, à la fois en ville et dans les faubourgs, une véritable polarisation de l'espace, qui oriente la localisation de l'ensemble des propriétés sénatoriales. Enfin, il faut souligner que les biens aristocratiques constituent souvent des oikoi, qui endossent une vocation publique, qu'elle soit religieuse, charitable ou balnéaire. Au cours des ve et $\mathrm{VI}^{\mathrm{e}}$ siècles, certaines des prérogatives impériales ont été ainsi de plus en plus assurées par les fortunes sénatoriales, dont I'empereur garde par ailleurs largement le contrôle. De la sorte, la structure des biens fonciers sénatoriaux aide à comprendre l'évolution qui conduit du Sénat romain à la cour impériale byzantine.
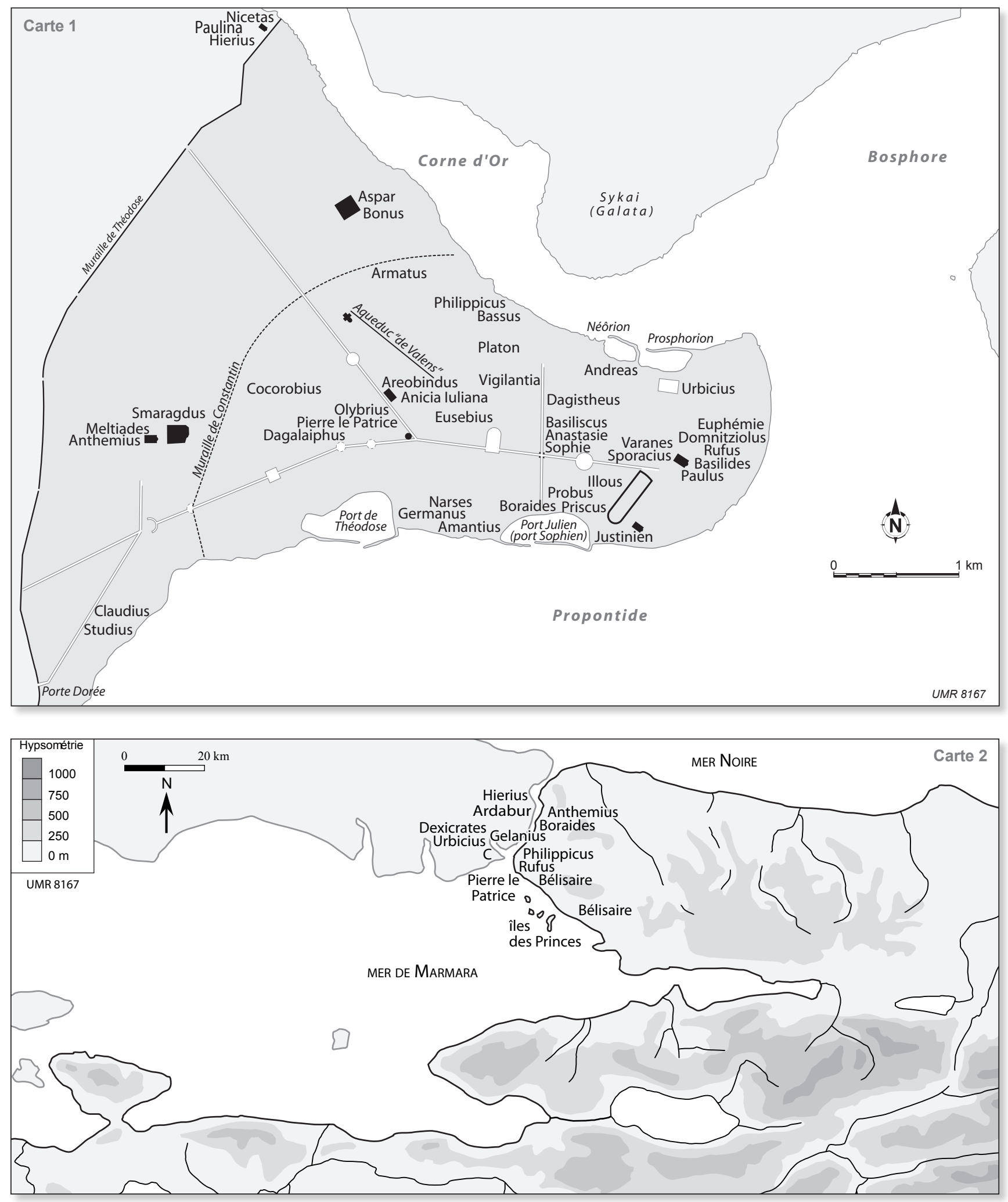


\section{ANNEXE PROSOPOGRAPHIQUE [59]}

\section{- Aetherius}

\section{PLRE IIIA : Aetherius 2}

Patrice, curator divinae domus Antiochi en 560-565. Gedeon 1893, p. $§ \bar{\sigma}{ }^{\prime}$ : borne de limite de propriété des

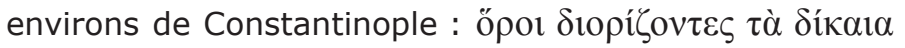

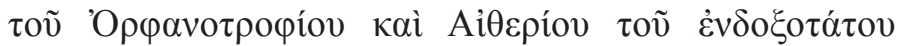

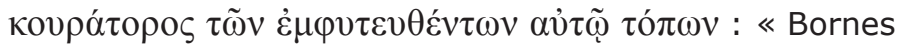
délimitant les biens de I'Orphelinat et d'Aetherius le très glorieux curateur des lieux dont il est emphytéote ».

Il est difficile de comprendre comment Aetherius pouvait détenir à bail emphytéotique une partie de la maison divine, sans doute celle d'Antiochus, dont il était curateur.

\section{- Amantius}

\section{PLRE II : Amantius 4}

Praepositus sacri cubiculi en 518.

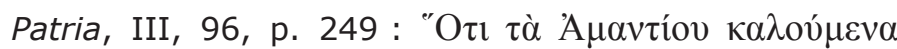

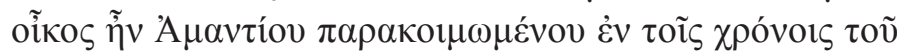

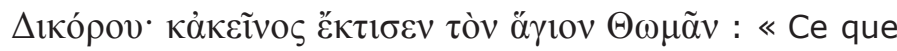
I'on appelle ta Amantiou était l'oikos du parakoimômenos Amantius au temps du Dikoros [Anastase] ; et celuilà bâtit l'église Saint-Thomas ».

Le terme de parakoimômenos pour qualifier Amantius est un anachronisme, mis pour praepositus sacri cubiculi.

Anthologie grecque, I, 5, p. 14 : «Tu as élevé à Dieu cette demeure (oĩkov), Amantius, au milieu de la mer, en luttant contre les flots toujours agités. Ni le Notos ni Borée n'ébranleront ton palais auguste que protège ce temple divin. Puisses-tu vivre de longs jours ; car tu as su à la Nouvelle Rome, en te lançant dans la mer, donner un éclat plus grand » (trad. P. Waltz).

Zonaras, Histoires, XIV, 5, 6, p. 266 (sur le règne de

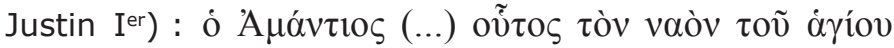

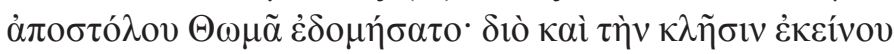

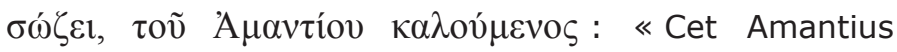
construisit le temple du saint apôtre Thomas : c'est pourquoi il préserve son nom, étant appelé d'Amantius ». JANIN 1964, p. 307-308; JANIN 1969, p. 248-250 ; BERGER 1988, p. 596-597 ( $n^{\circ} 181$ ).

L'église Saint-Thomas est située à l'ouest du port Sophien au bord de la mer.

\section{- Anastasie}

\section{PLRE IIIA : Aelia Anastasia (Ino) 2}

Épouse de Tibère II.

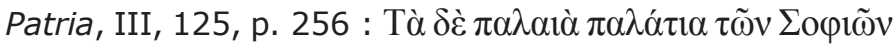

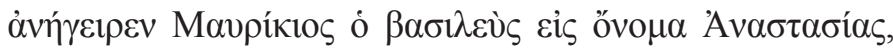

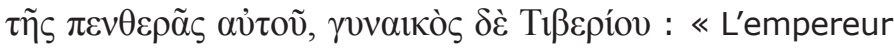
Maurice bâtit I'ancien palais de ta Sophiôn au nom de sa belle-mère Anastasie, épouse de Tibère ».

JANIN 1964, p. 308 ; BeRger 1988, p. 435-438 (n 99). Les Sophianai sont situés sur la Mésè près de ta Dareiou et de ta Basiliskou.

[59] Pour la plupart des personnages, on a renvoyé à sa notice dans la PLRE en respectant la façon de désigner le rang sénatorial: illustris, SPECTABILIS, CLARISSIMVS. Sauf exception, on n'a mentionné que les titres les plus élevés des personnages. Les dates des titres sont celles de leurs (premières) attestations : elles ne préjugent pas d'un port plus long par les personnages. Sont ensuite évoquées les sources concernant leurs biens fonciers; quand des sources médiévales répètent les données des sources antiques, elles ne sont en général pas indiquées ; dans quelques très rares cas les sources ne sont pas mentionnées car elles sont tardives et difficiles à interpréter (on a alors renvoyé à des études). Sauf mention contraire, toutes les traductions sont les nôtres. Viennent enfin des éléments bibliographiques et la localisation. 
- Anatolius

PLRE IIIA : Anatolius 7

Consul honoraire et curator domus divinae en 557.

Agathias, Histoires, V, 3, 11, p. 168 (séisme de 557 à Constantinople): «Cet Anatolius dormait dans sa

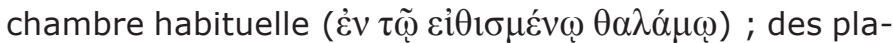
ques de marbre élégamment disposées étaient fixées à la cloison près de sa couche, telles qu'en font appliquer contre leurs murs, pour faire montre de leur luxe et de leur magnificence, ceux qui se plaisent outre mesure à des ornements superflus et inutiles. Une lourde plaque, arrachée et projetée par le séisme, tombe sur sa tête et la brisa (...) 》 (trad. Maraval 2007, p. 228).

\section{- Andreas}

PLRE II : ANDREAS LAUSIACUS 10

Cubicularius en 518.

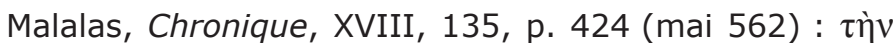

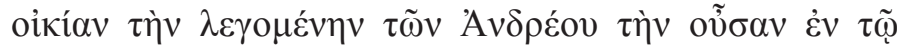

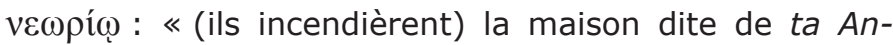
dreou située au Neôrion ».

La maison d'Andreas est située au Neôrion.

- Anicia Juliana

PLRE II : Anicia Iuliana 3

Épouse d'Areobindus.

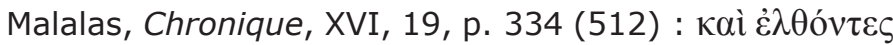

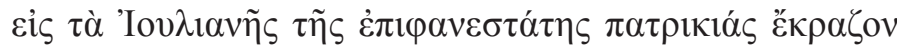

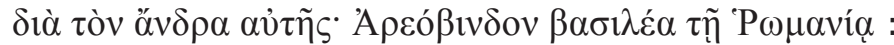
« et ils se rendirent chez Iuliana [à ta Ioulianès], patricienne très illustre, et chantèrent à l'intention de son mari : Areobindus empereur de l'État romain ».

JANIN 1964， p. 360-361; BERgER 1988， p. 510-512 (n०135).

Iuliana est la fondatrice de l'église Saint-Polyeucte, dont des ruines ont été retrouvées à droite de la Mésè quand on va du Philadelphion au forum de Marcien, à peu près à mi-chemin.

La maison de Iuliana était donc certainement située dans ce secteur, une localisation renforcée par deux autres indices. D'une part, l'oikos d'Olybrius, père de Iuliana, se situe entre le Philadelphion et les Saints-Apôtres ; or il s'y trouve une église Sainte-Euphémie commencée par sa belle-mère Eudoxia (fille de Théodose II), embellie par Olybrius et son épouse Placidia, puis par leur fille Anicia Iuliana. D'autre part, l'oikos d'Areobindus, époux de Iuliana, est situé dans le quartier des Constantinianae, au nord-ouest de Saint-Polyeucte, entre l'aqueduc de Valens et la Mésè.

\section{- Anthemius}

\section{PLRE II : Anthemius 3}

Maître des milices en 454-467, consul en 455, patrice en 455-467, empereur d'Occident en 467-472.

\section{Constantinople}

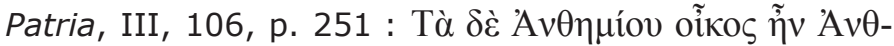

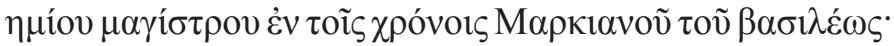

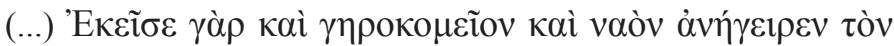

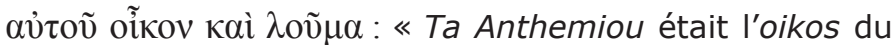
magister Anthemius au temps de I'empereur Marcien ; (...) là [à Constantinople] en effet il fit de son propre oikos avec son bain, un hospice et une église ».

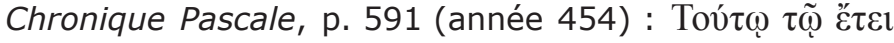

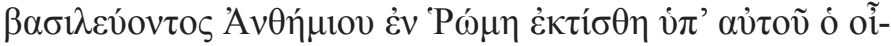

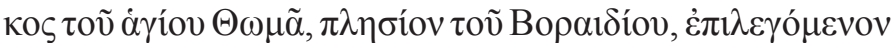

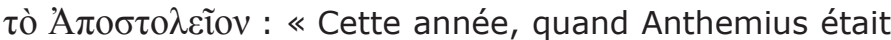
empereur à Rome, l'église de Saint-Thomas, appelée l'Apostoleion, fut fondée par lui près du Boraidion ».

Chronique Pascale, p. 598 (année 468, consulat d'Anthe-

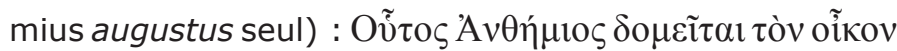

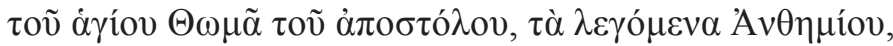

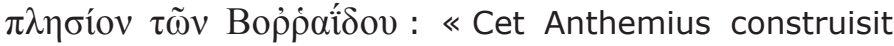
I'église de Saint-Thomas, l'apôtre, appelée celle d'Anthemius, près du quartier du Borraidon ».

JANIN 1964, p. 483 ; JANIN 1969, p. 251, 553 ; BERgER 1988 , p. 507-508 ( $n^{\circ} 132$ ).

L'église Saint-Thomas ta Anthemiou est située à l'ouest de l'église de Saint-Mokios, près de ta Olympiou et de ta Kyrou.

\section{Rive asiatique du Bosphore}

JANin 1964, p. 483.

Ta Anthemiou est situé sur la rive asiatique du Bosphore, à côté du Boraidion, près de Kanlica, à peu près à michemin entre Constantinople et la mer Noire.

\section{- Apion}

\section{PLRE IIIA : Fl. Strategius Apion Strategius Apion 3}

Consul en 539, patrice.

Malalas, Chronique, XVIII, 135, p. 423 (mai 562) : (...)

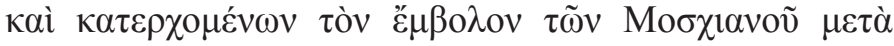

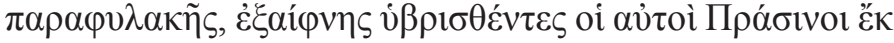

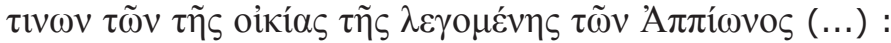
« [tandis que les Verts] descendaient le portique de Moschianus avec une garde, ils furent soudain insultés par des personnes venant de la maison dite ta Appiônos ». JANIN 1964, p. 311.

GAscou 1985, p. 188-190, 197 : sa qualité de protopatrice voulait qu'il préside le Sénat en tant que doyen d'âge et donc qu'il réside à Constantinople, ce qui concorde avec la mention de sa maison. 


\section{- Ardabur}

PLRE II : Ardabur iunior 1

Consul en 447, magister militum per Orientem vers 453 466, patrice au moins à partir de 453.

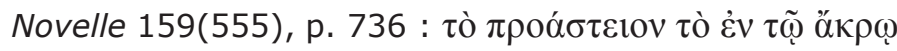

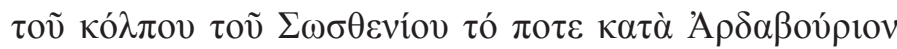

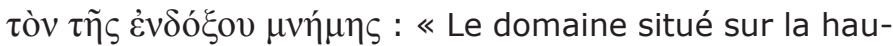
teur du golfe de Sôsthenion et qui appartenait auparavant à Ardabur de glorieuse mémoire ».

\section{- Areobindus}

\section{FI. Areobindus Dagalaiphus Areobindus 1}

Époux d'Anicia Iuliana.

Magister militum per Orientem vers 503-505, consul en 506.

Candidat au trône impérial de la foule chalcédonienne de Constantinople en 512.

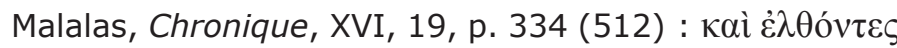

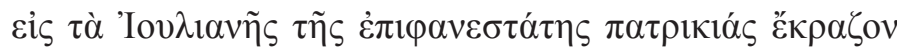

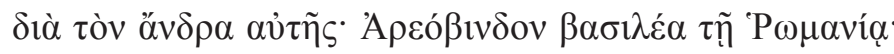
« et ils se rendirent chez Iuliana [à ta Ioulianès], patricienne très illustre, et chantèrent à I'intention de son mari : Areobindus empereur de l'État romain »; Areobindus fuit et se cache à Pérama (en face de Galata).

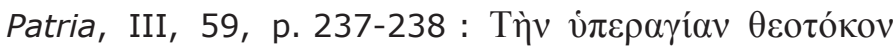

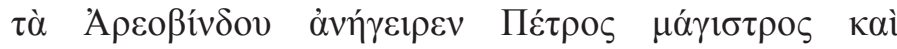

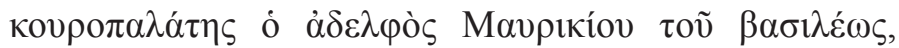

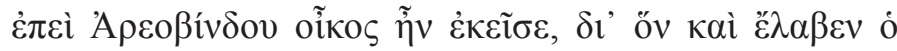

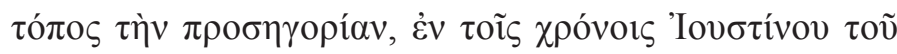

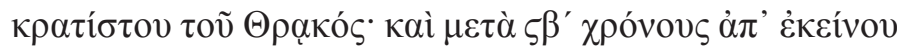

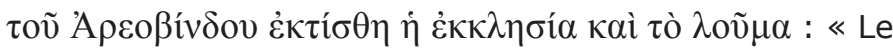
magister et curopalate Pierre, frère de l'empereur Maurice, bâtit l'église de la très sainte Théotokos dite ta Areobindou, car se trouvait là l'oikos d'Areobindus, duquel le lieu reçut aussi son appellation, au temps du puissant Justin le Thrace [Justin $\mathrm{I}^{\mathrm{er}}$ ] ; et 92 ans après feu Areobindus fut fondée une église avec un bain ».

Théophane, Chronographie, p. 277, évoque les constructions de Pierre frère de Maurice en 598, donc I'oikos d'Areobindus peut être placé 92 ans auparavant en 506, ce qui correspond exactement à la date de son consulat, et ne se situe donc pas sous Justin Ier $^{\text {mais }}$ sous Anastase.

JANIN 1964, p. 313-314; Janin 1969, p. 157 ; Berger 1988 , p. 512-513 ( $n^{\circ} 137$ ).

L'oikos d'Areobindus est situé dans le quartier des Constantinianae, au nord-ouest de Saint-Polyeucte, entre I'aqueduc de Valens et la Mésè.

\section{- Armatus}

PLRE II : Armatus

Neveu de Vérine (épouse de Léon I ${ }^{\text {er }}$ et sœur de Basiliscus)
Magister militum praesentalis vers 475, consul en 476, patrice.

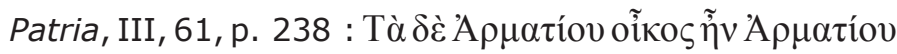

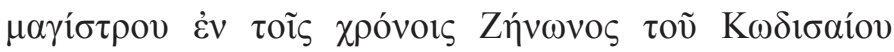

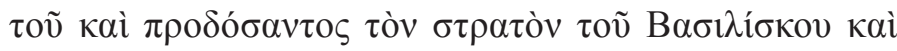

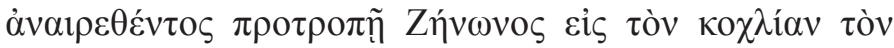

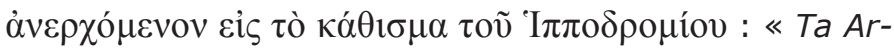
matiou était l'oikos du magister Armatius au temps de Zénon Kôdisaios ; il trahit l'armée de Basiliscus et, sur ordre de Zénon, fut mis à mort dans le konchlion (escalier en colimaçon) qui conduit au kathisma (loge) de I'hippodrome ».

D'autres sources confirment la trahison de Basiliscus par Armatus mais pas la mise à mort de ce dernier dans de telles conditions.

Janin 1964, p. 314 ; Janin 1969, p. 157 ; Berger 1988, p. 497-499 ( $\left.n^{\circ} 127\right)$.

L'église de la Théotokos ta Armatiou avec I'hospice et le bain remontent au règne de Maurice.

L'oikos d'Armatus est situé au sud-est de la citerne d'Aspar, de l'autre côté de la muraille de Constantin.

\section{- Aspar}

\section{PLRE II : FI. Ardabur Aspar}

Magister militum en 431-471, consul en 434, patrice en 451.

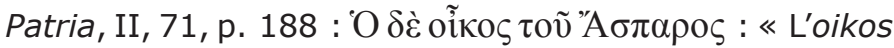
d'Aspar ».

J ANin 1964, p. 316 ; Berger 1988, p. 611-613 ( $n^{\circ} 188$ ). Si la citerne d'Aspar est très bien localisée, il n'est pas évident a priori que l'oikos du même personnage ait été situé dans le même secteur. Néanmoins les cas de Bonus et Illous, détenteurs d'un oikos et éponymes d'une citerne, tendent à montrer que les localisations convergent, d'autant qu'il est probable que la citerne de Bonus était anciennement celle d'Aspar : en fut-il de même pour l'oikos?

\section{- Basilides}

\section{PLRE IIIA : Basilides}

Préfet du prétoire d'Orient en 528, patrice en 528, préfet du prétoire d'Illyricum en 529, quaestor sacri palatii en 532, maître des offices en 536-539, consul honoraire en 536 .

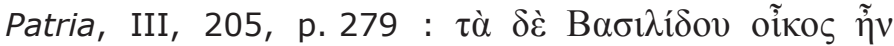

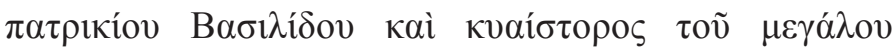

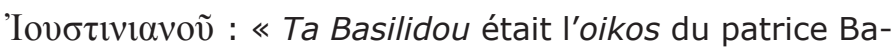
silides questeur de Justinien le Grand ».

JANIN 1964, p. 298-299, 318 ; BERGER 1988, p. 430-432 (n॰ 97).

L'oikos de Basilides est situé immédiatement à l'est de Sainte-Sophie. 


\section{- Basiliscus}

\section{PLRE II : Fl. Basiliscus 2}

Frère de Vérine (épouse de Léon $\mathrm{I}^{\mathrm{er}}$ )

Magister militum vers 464-468, consul en 465, patrice, empereur en 475-476.

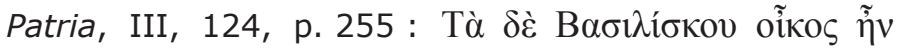

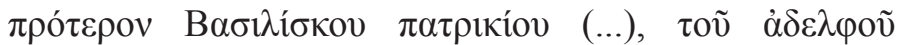

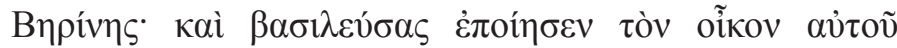

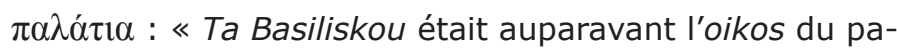
trice Basiliscus (...), frère de Vérine ; et, quand il devint empereur, il transforma l'oikos en palais ».

JANin 1964, p. 123, 319-320 ; Berger 1988, p. 433-435 (n०99).

L'oikos de Basiliscus se situe au nord-ouest du forum de Constantin.

\section{- Bassus}

\section{PLRE IIIA : Bassus 2}

Patrice sous Justinien.

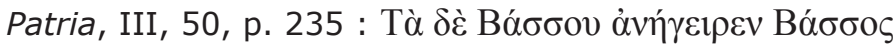

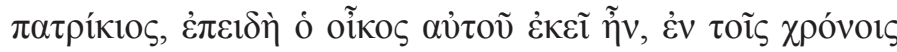

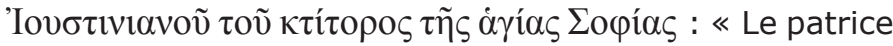
Bassus bâtit ta Bassou, car son propre oikos se trouvait là, au temps de Justinien, le constructeur de Sainte-Sophie ».

Janin 1964, p. 320 ; Berger 1988, p. 456-458 (n 108). L'oikos de Bassus est situé à l'est du Zeugma, entre ta Karpianou et Oxeia.

\section{- Bélisaire}

\section{PLRE IIIA : FI. Belisarius 1}

Magister militum per Orientem 529-551, patrice, consul en 535.

\section{Panteichion}

Procope, Guerre contre les Goths, III, 35, 4 (533) : ฑ๊v

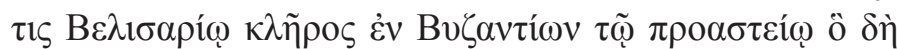

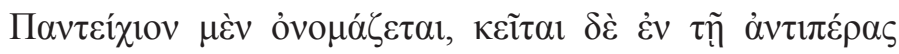

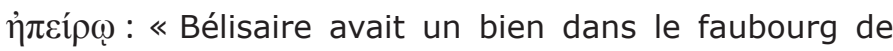
Byzance qui porte le nom de Panteichion et qui est situé sur la rive opposée ».

Cette terre se composait au moins de vignes. Selon Procope, leur raisin aurait produit du vin en excès, ce qui passait pour un présage des futurs succès de Bélisaire avant qu'il ne s'embarque contre les Vandales en 533.

Bélisaire possédait en 533 une propriété héritée ( $\kappa \lambda \tilde{\eta} \rho \varsigma)$ à Panteichion sur la rive asiatique du Bosphore.

\section{Rufinianae}

Procope, Guerre contre les Perses, I, 25, 21 (541) : Procope évoque en ces termes un trajet de l'épouse de Béli-

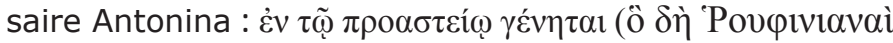

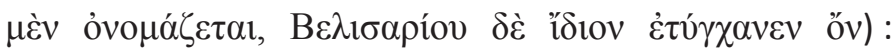
«Elle est arrivée dans le domaine (appelé Rufinianae qui était le bien privé de Bélisaire) ».

Bélisaire possédait en 541 le domaine des Rufinianae, proche de Panteichion.

\section{Maison urbaine}

Marcellinus comes, Chronique, add. a. 544 : In Oriente Belisario constituto exulatur Iohannes ex consule ordinario patricius atque praefectus praetorio et domus eius datur Belisario : «Quand Bélisaire était installé en Orient, Jean le patrice, ex consul ordinaire et préfet du prétoire fut exilé et sa maison fut donnée à Bélisaire ». JANIN 1964, p. 136, 502.

\section{- Bonus}

\section{PLRE IIIA : Bonus 5}

Patrice en 622-626.

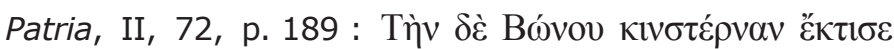

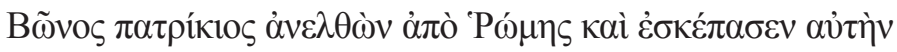

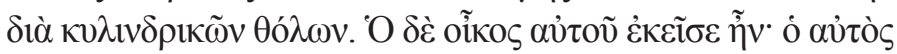

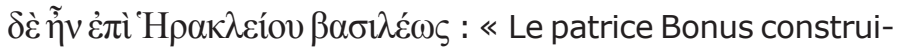
sit la citerne de Bonus quand il vint de Rome et il la couvrit de voûtes cylindriques. Son propre oikos se trouvait là ; il vivait lui-même sous l'empereur Héraclius ».

La mention d'une venue de Bonus de Rome à Constantinople est douteuse.

JANin 1964, p. 128-129, 206-207 ; Berger 1988, p. 613615 ( $n^{\circ} 189$ ).

La citerne de Bonus est probablement à identifier à celle d'Aspar ; Bonus se serait alors borné à la couvrir de la manière qu'indiquent les Patria.

\section{- Boraides}

PLRE IIIA : Boraides

Cousin de Justinien, frère de Germanus.

\section{Constantinople}

Jean d'Antioche, fr. Roberto, p. 552, I. 21-23 (4 oc-

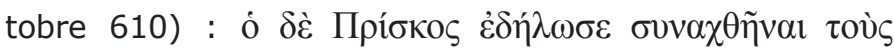

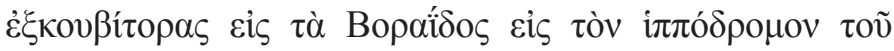

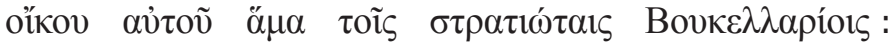
«Priscus commit le méfait de rassembler les excubitores avec les soldats bucellaires à ta Boraidos dans I'hippodrome de son oikos ».

JANIN 1964, p. 325-326.

Ta Boraidos est situé au nord du port Sophien.

2. Rive asiatique du Bosphore

JANin 1964, p. 484

Le Boradion est situé sur la rive asiatique du Bosphore, près de Kanlica, à peu près à mi-chemin entre Constantinople et la mer Noire. 


\section{- Claudius}

Patrice et questeur sous Basiliscus.

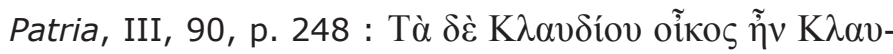

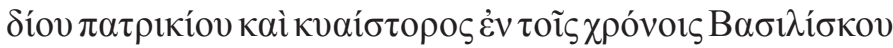

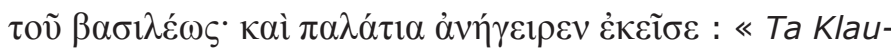
diou était l'oikos du patrice Claudius, questeur au temps de I'empereur Basiliscus ; et en ce lieu il bâtit un palais ».

JANIN 1964, p. 370-371 ; Berger 1988, p. 593 ( $n^{\circ} 179$ ) et p. 605-606 ( $n^{\circ} 185$ ).

L'oikos de Claudius est situé à Psamathia près du Stoudios.

\section{- Cocorobius}

PLRE IIIA : Ioannes qui et Coccorobius 45

Préfet de Constantinople en 550.

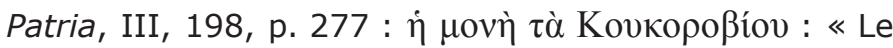
monastère ta Koukorobiou ».

Janin 1964, p. 371 ; Berger 1988, p. 631-632 ( $n^{\circ} 200$ ). Le monastère, d'époque médiévale, est situé dans la vallée du Lycus, proche du monastère de Libs, ce qui permet de localiser les biens de Ioannes Cocorobius.

\section{- Dagalaiphus}

\section{PLRE II : FI. Dagalaiphus 2}

Père de Fl. Areobindus Dagalaiphus Areobindus 1.

Consul en 461, patrice en 475/476.

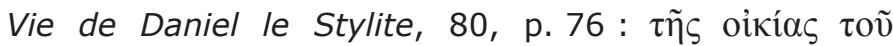

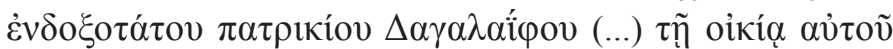
$\pi \lambda \eta \sigma i ́ o v$ oõ Boó $\varsigma$ : «La maison du gloriosissime patrice Dagalaiphus (...) sa maison près du Forum du Bœuf » (trad. Festugière 1961, p. 149).

La maison de Dagalaiphus est située près du forum du Bœuf.

\section{- Dagisthaeus}

\section{PLRE IIIA : Dagisthaeus 2}

Magister militum per Armeniam en 548-549.

Malalas, Chronique, XVIII, 17, p. 364 (518) : 'O $\delta \dot{\varepsilon}$

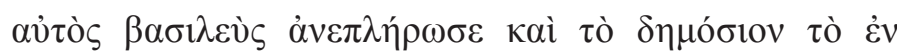

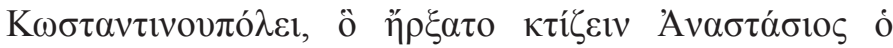

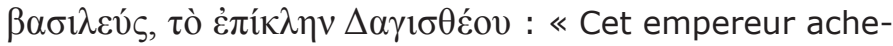
va le bain public situé à Constantinople que l'empereur Anastase avait commencé à construire et qui était dit de Dagistheus ».

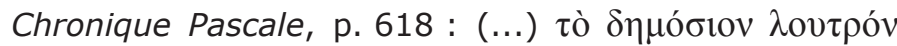

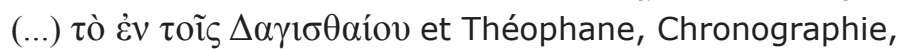

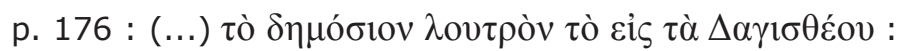
« Le bain public situé à ta Dagistheou ».

JANin 1964, p. 331 ; Berger 1988, p. 439-442 ( $n^{\circ} 101$ ). Ta Dagistheou est situé entre les forums de Constantin et Théodose sur le portique de Domninos perpendiculaire à la Mésè, vers le milieu du côté Est.
Dagistheus n'est pas le fondateur du bain mais possédait un bien à proximité.

Le maître des milices Dagisthaeus est attesté après le début de la construction du bain par Anastase, mais il a donné son nom au quartier, qui peut très bien être ainsi désigné par Malalas, la Chronique Pascale et Théophane. L'attribution du quartier à I'otage goth Dagistheus, attesté en 479, n'est pas plausible, d'autant que l'une des sources, la Chronique Pascale, emploie la forme $\Delta \alpha \gamma 1 \sigma \theta \alpha i ́$ s, qui est utilisée par Procope pour le maître des milices. Il reste que ce dernier peut être le fils de l'otage goth.

\section{- Dexicrates}

\section{PLRE II : FI. Dexicrates}

Patrice, consul en 503.

AvramÉA 1987, p. 399-401: plaque de marbre découverte dans la banlieue européenne de Constantinople,

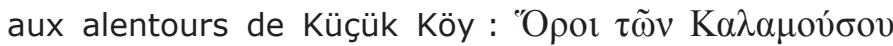

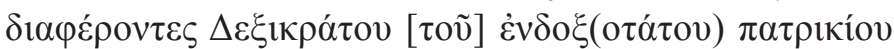

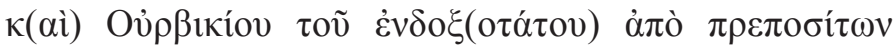
$[\dot{\alpha}] \pi$ ò vं $\alpha \tau \omega v$ : «Bornes de ta Kalamousou appartenant à Dexicrates, le très glorieux patrice, et à Ourbikios, le très glorieux ex-préposite, ex-consul ».

Nous ignorons quel est le domaine appelé ta Kalamousou.

\section{- Domnitziolus}

\section{PLRE IIIA : Domnitziolus 2}

Neveu de Phocas.

Patrice et curopolate en 603-610, magister militum per Orientem en 604.

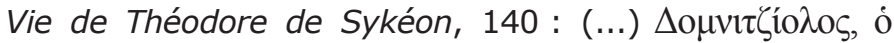

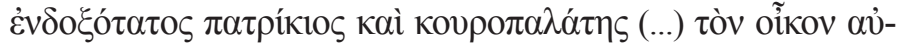

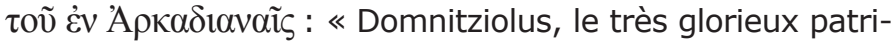
ce et curopalate (...) I'oikos de celui-ci aux Arcadianae ». JANIN 1964, p. 292-293.

L'oikos de Domnitziolus est situé aux Arcadianae.

\section{- Eugenius}

\section{PLRE IIIA : Eugenius 1}

Préfet du prétoire d'Orient en 548.

Théophane, Chronographie, p. 235 (560) : Ev̉yćvios ó

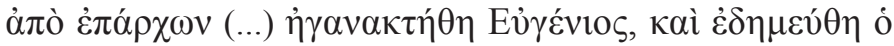
oĩkos $\alpha$ นoṽ : «L'ex préfet Eugenius (...) Eugenius fut disgracié et son oikos fut confisqué ».

\section{- Eulogius}

\section{PLRE II : Eulogius 9}

Préfet du prétoire honoraire et patrice en 525-532.

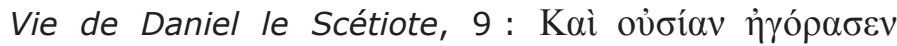

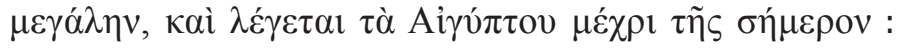
« Il acheta une vaste propriété, qui est appelée ta Aigyptou jusqu'à nos jours ». 
Ce personnage, protagoniste de la sédition Nika en 532, n'est étrangement connu que par cette source hagiographique, mais la précision des données incite à retenir son existence.

DAGRON 1977, p. 8.

\section{- Euphémie}

PLRE II : Euphemia 3

Sœur de Léon Ier.

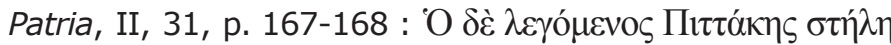

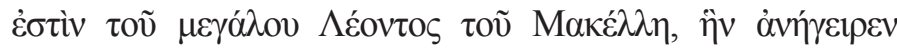

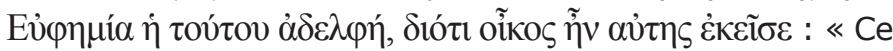
que I'on appelle le Pittakès est une statue du Grand Léon le boucher qui a été élevée par sa sœur Euphémie, parce que l'oikos de celle-ci se trouvait là ».

La statue de Léon Ier s'élevait aux Pittakia mais ce n'est pas elle qui est à l'origine du nom du quartier.

JANIN 1964, p. 350, 413 ; BERGER 1988, p. 394-396 (n॰ 81). Les Pittakia sont situés au nord-est de Sainte-Sophie et de Sainte-Irène, sur la rue qui va de l'augustaion à l'acropole.

\section{- Eusebius}

\section{PLRE IIIA : Eusebius 6}

Patrice sous Justin II.

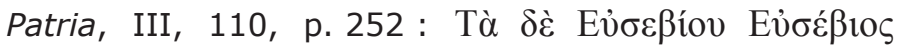

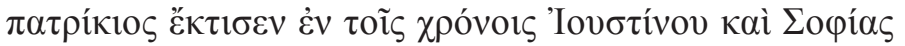

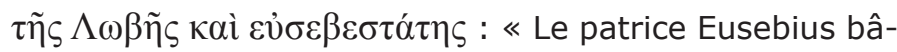
tit ta Eusebiou au temps de Justin et de Sophie honteuse et pieuse ».

Janin 1964, p. 351 ; Berger 1988, p. 509 ( $n^{\circ} 133$ ).

Les biens d'Eusebius sont situés à proximité de ta Kyrou, au nord-ouest du forum de Théodose.

\section{- Gelanius}

PLRE II : GELANIVS

Castrensis en 460.

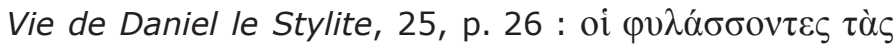

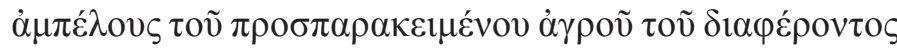

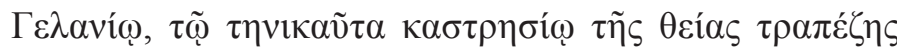

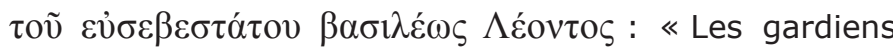
des vignes d'un champ voisin appartenant à Gelanius, alors intendant de la table sacrée du très pieux empereur Léon » (trad. Festugière 1961, p. 108-109).

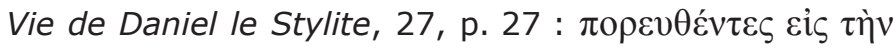

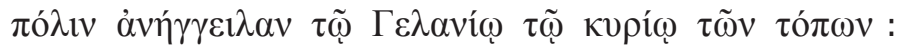
« [Ils] allèrent à la ville annoncer la chose à Gelanius, propriétaire de ces lieux » (trad. Festugière 1961, p. 110).

Le domaine comprend des vignes ; Gelanius habite en ville. JANIN 1964, p. 468.

Le domaine est situé à Anaplous, juste au nord de Constantinople sur la rive européenne du Bosphore (à Arnavutköy).

\section{- Germanus}

\section{PLRE II : Germanus 4}

Cousin de Justinien, frère de Boraides.

Magister militum praesentalis, consul honoraire et patrice en 536-550.

ACO, IV, 1, p. 30 (553) : Paulum religiosissimum episcopum secundae Iustinianae, qui habitat in domo Germani prope portum Caesarii : «Paul le très religieux évêque de Justiniana Secunda qui habite dans la maison de Germanus près du port Césarien ».

Janin 1964, p. 354 ; Berger 1988, p. 746-747.

La maison de Germanus est proche du port Césarien. Elle se situe donc à proximité des biens de son frère Boraides, dont il a hérité. Il faut noter que Germanus est mort en 550, trois avant la visite de l'évêque de Iustiniana Prima, une cité d'Illyricum, région dont était originaire le cousin de Justinien.

\section{- Hierius}

PLRE II : Hierius 7 (père), Alexander 19, Anthemius 8, CALLIOPIVS 4 et Constantinus 17 (fils); Hierius 6 ; CALLIOPIVS 3.

Patrice et préfet du prétoire d'Orient en 494-496.

Le propriétaire Hierius peut être identifié à Hierius patrice et préfet du prétoire d'Orient en 494-496 pour plusieurs raisons : ils ont tous deux un parent Calliopius (fils pour le premier, parent pour le second) ; le fils du premier est titré clarissimus alors que le parent du second fut nommé par Hierius comes Orientis (une charge qui correspond au rang de clarissimus); la famille du premier possède aussi une maison à Antioche (transmise à son autre fils Constantinus), qui appartiendrait donc au frère d'un comes Orientis ; la famille du premier possède un proasteion appartenant auparavant à Ardabur, également possessionné à Antioche ; un Alexander fils de Hierius était associé à un groupe de patrices chargé à Beroia de la défense face aux Perses en 528 ; I'ampleur de la fortune constantinopolitaine est du niveau d'un patrice et préfet du prétoire. Il reste à se demander pourquoi la Novelle 159 ne reprend pas les titres des personnages : il est possible que les fonctions détenues sous Anastase n'aient pas été rappelées dans un texte datant de Justinien.

Novelle 159 (555), p. 736, I. 17-31 : Le testament du

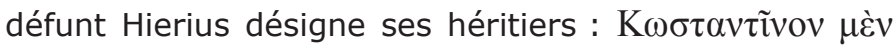

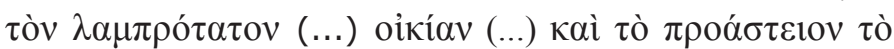

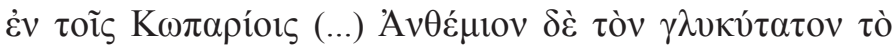

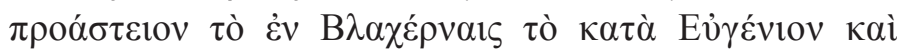

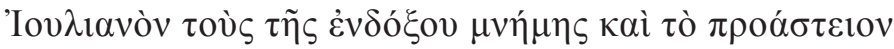

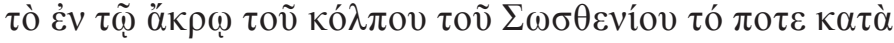

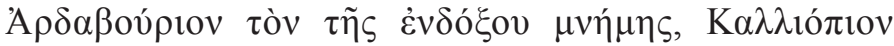

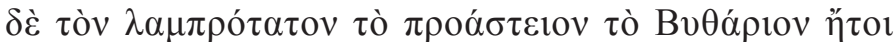




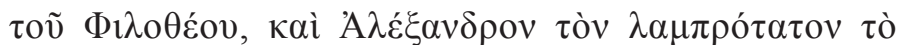

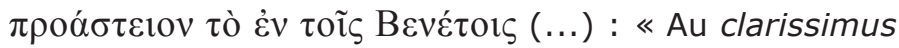
Constantin (...) une maison (...) et le domaine situé à ta Kôparia (...) Au très heureux Anthemius le domaine situé aux Blachernes qui appartenait à Eugenius et Iulianus de glorieuse mémoire et le domaine situé sur la hauteur du golfe de Sôsthenion qui appartenait auparavant à Ardabur de glorieuse mémoire. Au clarissimus Calliopius le domaine de Bytharion autrement dit de Philothée. Et au clarissimus Alexandre le domaine des Benetoi ».

L'un des domaines se trouve à Sôsthenion, au centre du Bosphore, côté européen.

JANIN 1964, p. 479.

Novelle 159 (555), p. 738, I. 1-12 : bien légué par Hierius à son fils Constantinus et au fils de ce dernier le clarissimus Hierius :

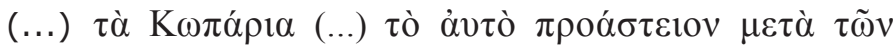

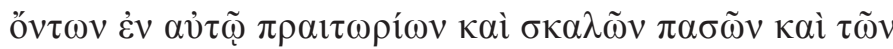

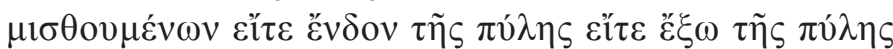

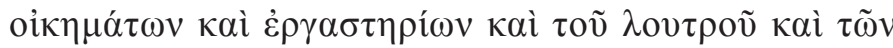

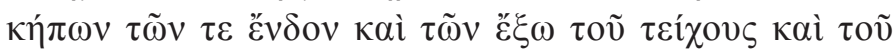

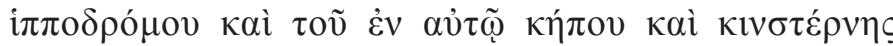
(...) : «Ta Kôparia (...) ce domaine avec les palais qui s'y trouvent et tous les ports, les locaux et ateliers loués à l'intérieur comme à l'extérieur de la porte, les bains, les jardins situés dans l'enceinte comme en dehors, I'hippodrome, ainsi que le jardin qu'il renferme, la citerne 》.

KAPLAN 1992, p. 147-148.

\section{- Illous}

PLRE II : Illus 1

Maître des offices en 477-481, patrice en 477, consul en 478, magister militum per Orientem en 481-483.

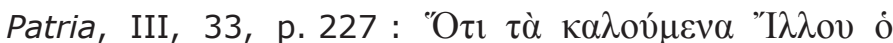

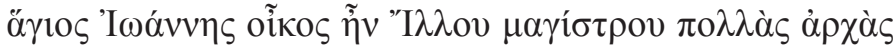

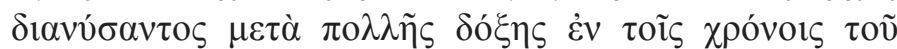

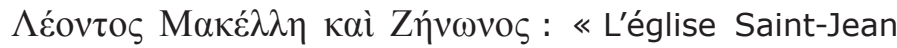
dénommée ta Illou était l'oikos du magister Illus qui occupa de nombreux postes de pouvoir avec une grande gloire au temps de Léon le boucher et de Zénon ».

Une basilique a été reconstruite par Illous en 478 : Jean

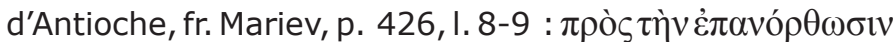

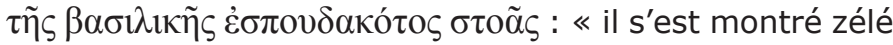
dans la restauration de la stoa basilikè ».

Théophane, Chronographie, p. 176, affirme à propos de

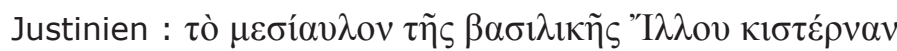
$\mu \varepsilon \gamma \alpha \dot{\alpha} \lambda \eta v \dot{\varepsilon} \pi$ oín$\sigma \varepsilon v$ : «il transforma en grande citerne la cour intérieure de la basilique d'Illus ».

JANIN 1964, p. 37, 157, 176-177; Janin 1969, p. 416 ; BERGER 1988, p. 559-562 ( $\left.\mathrm{n}^{\circ} 161-162\right)$.

L'église Saint-Jean ta Illou était située entre le forum de
Constantin et l'hippodrome. Or cette localisation est précisément celle de la citerne-basilique. Donc la basilique construite par Illous était certainement une construction présente sur une partie de son oikos.

\section{- Jean de Cappadoce \\ PLRE IIIA : FI. Ioannes 11}

Préfet du prétoire d'Orient en 531-541, consul honoraire et patrice en 535, consul en 538. Marcellinus comes, Chronique, add. a. 544 : In Oriente Belisario constituto exulatur Iohannes ex consule ordinario patricius atque praefectus praetorio et domus eius datur Belisario: «Quand Bélisaire était installé en Orient, Jean le patrice, ex consul ordinaire et préfet du prétoire fut exilé et sa maison fut donnée à Bélisaire ».

\section{- Justinien}

\section{PLRE II : Fl. Petrus Sabbatius Iustinianus 7}

Neveu de Justin Ier.

Magister militum praesentalis en 520-527, consul en 521, patrice.

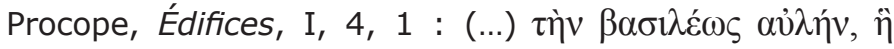

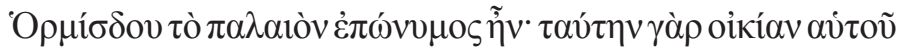

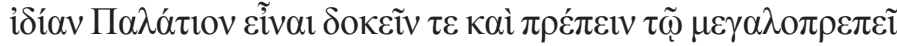

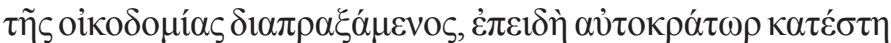

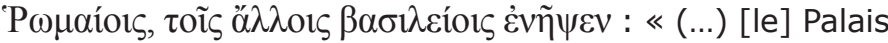
impérial qui jadis portait le nom d'Hormisdas. Justinien s'était employé à ce que cette résidence, dont il avait fait sa demeure personnelle, passât pour un palais et se distinguât par la magnificence de sa construction, et quand il était devenu empereur des Romains, il l'avait rattachée aux autres palais » (trad. Roques 2011, p. 85).

Janin 1964, p. 137 ; BerGer 1988, p. 566-568 (n 165). Le palais de Justinien est l'ancien palais d'Hormisdas, à I'est du port Sophien.

\section{- Marcellus}

\section{PLRE IIIB : Marcellus 5}

Frère de Justin II.

Maître des milices en 544, patrice en 565.

Jean d'Éphèse, Histoire ecclésiastique, III, 5, 18 : « La totalité des biens du grand patrice Marcellus, frère du défunt empereur Justin, qui n'était pas moins grande que les domaines impériaux, c'est-à-dire ses maisons et ses biens fonciers, l'or et l'argent, la garde-robe, et tous les biens dont il disposait partout sans exception » (trad. C. Fauchon).

\section{- Marinus}

\section{PLRE II : Marinus 7}

Préfet du prétoire sous Anastase.

Marcellinus comes, Chronique, a. 512 : sa maison (domus) est incendiée lors de l'émeute. 


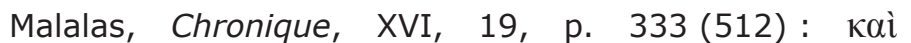

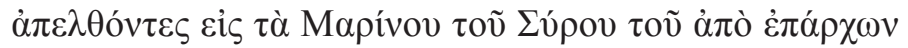

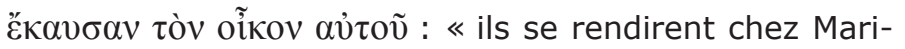
nus [à ta Marinou] le Syrien l'ex préfet et incendièrent son oikos ».

JANIN 1964, p. 385.

\section{- Maxentianus}

\section{PLRE IIIB : Maxentianus}

Magister militum praesentalis et consul honoraire en 536. ACO, IV, 1, p. 30 (553) : Proiectum reuerentissimum episcopum Naisitanum Illyricae dioceseos, qui habitat in domo Maxentiani : «Proiectus très révérend évêque de Naissus dans le diocèse d'Illyricum, qui habite dans la maison de Maxentianus ».

\section{- Meltiades}

PLRE IIIB : Meltiades

JANIN 1964, p. 389-390; JANIN 1969, p. 493-494; FEISSEL 2005, p. 252-256.

Ta Meltiadou est situé entre la citerne de Saint-Mokios et la muraille de Théodose. Ce nom dérive certainement des biens de Meltiades, que I'on peut identifier au grand personnage au service duquel se trouve le musicien Ablabius, impliqué dans le complot contre Justinien de 562 .

\section{- Musonius}

PLRE IIIB : Musonius 1 et Musonius 2

Préfet de Constantinople en 556.

Anthologie grecque, VIII, 677, p. 135 :

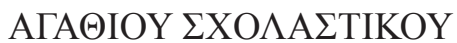

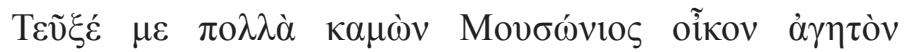

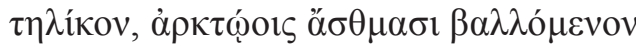

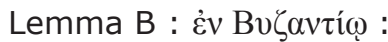

« D'Agathias le Scholastique

Celui qui m'a bâtie, à grand'peine, c'est Musonius : je suis une maison admirable, pas bien grande, mais exposée au souffle des vents du Nord.

À Byzance » (trad. P. Waltz \& G. Soury).

L'emploi d'oikos au lieu d'oikia a certainement des raisons métriques dans le vers d'Agathias.

Musonius 1 (préfet en 556) et Musonius 2 (bâtisseur de l'oikos) sont certainement les mêmes personnages, d'autant que I'auteur de l'épigramme est I'historien Agathias dit le Scholastique, qui est attesté comme étudiant en droit à Alexandrie en 551 et écrivit son ouvrage sous Justin II et Tibère II, donc entre 565 et 582 .

\section{- Narses}

\section{PLRE IIIB : Narses 1 et Narses 3}

Praepositus sacri cubiculi en 537/538-554, patrice en 559, consul honoraire en 565.

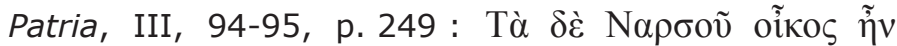

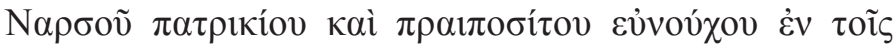

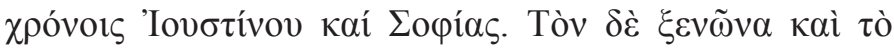

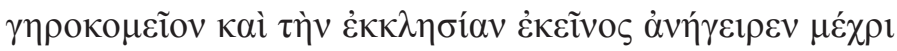

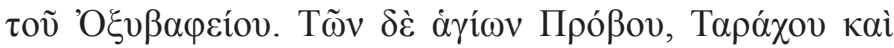

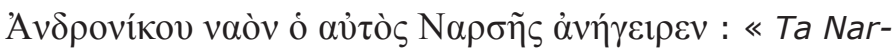
sou était I'oikos de l'eunuque Narses, patrice et praepositus au temps de Justin et Sophie. Il bâtit I'hôtellerie, I'hospice pour vieillards et l'église, jusqu'à I'Oxybapheion. Le même Narses bâtit l'église des Saints-Probus-Tarachus-et-Andronicus ».

Le propriétaire de l'oikos (PLRE IIIB : Narses 3) est certainement identique au patrice et praepositus sacri $\mathrm{Cu}$ biculi sous Justinien (PLRE IIIB : Narses 1 ) et les Patria font manifestement erreur en le plaçant sous Justin II. JANin 1964, p. 395 ; Janin 1969, p. 422-423 ; BERger 1988 , p. 593-595 ( $n^{\circ} 180$ ).

L'oikos de Narses est situé entre le forum de Théodose et la mer de Marmara.

\section{- Nicetas}

\section{PLRE IIIB : Nicetas 7}

Patrice en 610, comes excubitorum en 612-613.

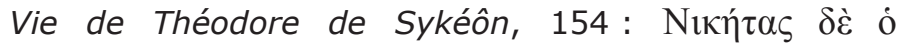

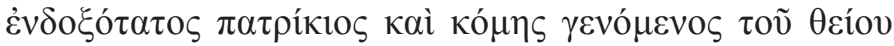

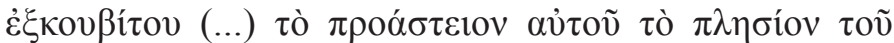

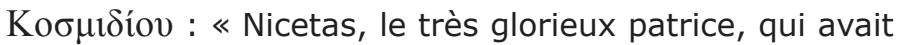
été fait comte des divins excubitores (...) son domaine près du Cosmidion » (trad. A.-J. Festugière).

\section{- Olybrius}

\section{PLRE II : Anicius Olybrius 6}

Consul en 464, empereur d'Occident en 472.

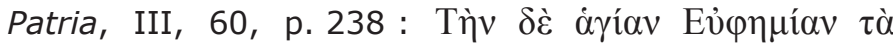

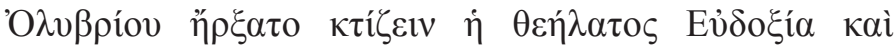

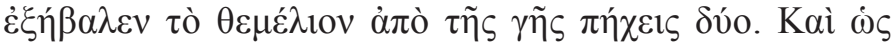

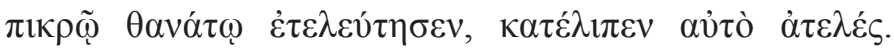

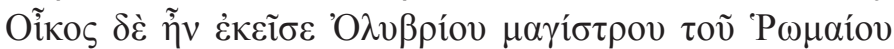

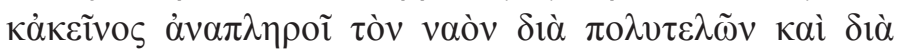

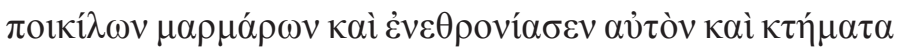

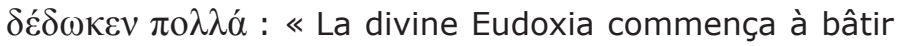
Sainte-Euphémie ta Olybriou et en éleva les fondations jusqu'à deux coudées à partir du sol. Et comme elle décéda d'une mort cruelle, elle laissa l'ouvrage inachevé. Là se trouvait I'oikos du magister Olybrius le Romain et celui-ci acheva l'église avec des marbres de nombreuses couleurs et variés et l'inaugura et lui donna de nombreux biens ».

On ne voit pas à quoi renvoie le titre de magister dans la carrière d'Olybrius.

Anthologie grecque, I, 12, p. 18 : « Je suis la demeure ( $\delta$ ó $\mathrm{o}_{\text {) }}$ de la Trinité, et trois générations m’ont faite. La première qui, échappée aux guerres et aux hordes 
barbares, m'a élevée et consacrée à Dieu comme rançon de ses épreuves, c'est la fille de Théodose, Eudoxia ; après elle, sa fille Placidia m'a ornée avec son bienheureux époux ; et l'éclat qui pouvait encore manquer à ma beauté, la généreuse Julienne me I'a donné en souvenir de ses parents : elle a porté très haut la gloire de sa mère, de son père et de l'illustre mère de sa mère, en embellissant encore mon ancienne parure pour créer une œuvre nouvelle » (trad. P. Waltz).

L'église Sainte-Euphémie a été commencée par sa belle-mère Eudoxia (fille de Théodose II), embellie par Olybrius et son épouse Placidia, puis par leur fille Anicia Iuliana.

JANIN 1964, p. 398-399; JANIN 1969, p. 124-126; Berger 1988, p. 496-497 ( $\left.n^{\circ} 126 a\right)$.

L'oikos d'Olybrius est situé entre le Philadelphion et les Saints-Apôtres.

\section{- Paulina}

Épouse de Zénon (PLRE II : FI. Zenon 6) : consul en 448, magister militum per Orientem en 447-451, patrice en 451.

Théophane, Chronographie, p. 380 : غ่v $\tau \tilde{\omega} v \alpha \tilde{\omega} \tau \tilde{\omega} v$

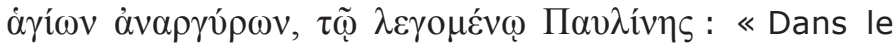
temple des Saints-Anargyres, dit de Paulina ».

JANIN 1964, p. 463 ; JANIN 1969, p. 286-289; BERGER 1988 , p. 670-673 ( $\left.n^{\circ} 226\right)$; FEISSEL 1999.

Ta Paulinès est situé au Cosmidion, hors les murs, après les Blachernes sur la Corne d'Or.

\section{- Paulus}

\section{PLRE IIIB : Paulus 23}

Père de Maurice.

Patrice.

Jean d'Éphèse, Histoire ecclésiastique, III, 5, 18 : « Et ensuite [Maurice] plaça son père à la tête du Sénat et chef de tous les patrices et lui offrit ainsi qu'à son fils Petrus, le frère de l'empereur, la totalité des biens du grand patrice Marcellus, frère du défunt empereur Justin, qui n'était pas moins grande que les domaines impériaux, c'est-à-dire ses maisons et ses biens fonciers, l'or et l'argent, la garde-robe, et tous les biens dont il disposait partout sans exception. Et ensuite, il donna à son père et à sa mère une autre maison près de l'église (de Sainte-Sophie) et de son propre palais » (trad. C. Fauchon).

\section{- Petrus}

PLRE IIIB : Petrus 55

Frère de Maurice.

Maître des milices en 593-602, curopalate en 602.

Jean d'Éphèse, Histoire ecclésiastique, III, 5, 18 :
«Et ensuite [Maurice] plaça son père à la tête du Sénat et chef de tous les patrices et lui offrit ainsi qu'à son fils Petrus, le frère de l'empereur, la totalité des biens du grand patrice Marcellus, frère du défunt empereur Justin, qui n'était pas moins grande que les domaines impériaux, c'est-à-dire ses maisons et ses biens fonciers, I'or et l'argent, la garde-robe, et tous les biens dont il disposait partout sans exception » (trad. C. Fauchon).

\section{- Philippicus}

\section{PLRE IIIB : Philippicus 3}

Époux de Gordia, soeur de Maurice.

Maître des milices en 584-614, patrice en 603.

1.Constantinople

Jean d'Éphèse, Histoire ecclésiastique, III, 5, 18 : «Peu après, il donna à sa sœur et à son mari Philippicus une maison vaste et solidement bâtie désignée d'Aloros [Hilara] dans la partie occidentale de la ville dans le faubourg appelé Zeugma » (trad. C. Fauchon).

La maison d'Hilara porte certainement le nom d'une femme de rang sénatorial dont Justinien s'appropria les biens (Procope, Histoire secrète, XII, 5).

Magdalino 1996, p. 43-44 et 46.

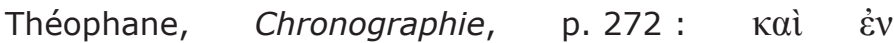

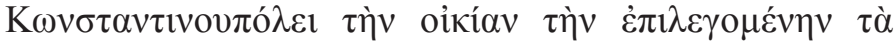
$\Phi \imath \lambda \imath \pi \pi \imath \kappa o \tilde{~: ~ « ~ E t ~ a ̀ ~ C o n s t a n t i n o p l e, ~ i l ~ b a ̂ t i t ~ l a ~ m a i s o n ~ a p-~}$ pelée ta Philippikou ».

Cette maison est sans doute différente de celle d'Hilara, qui n'a pas été construite par Philippicus.

JANIN 1964, p. 410.

2.Chrysopolis

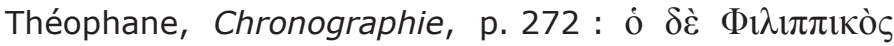

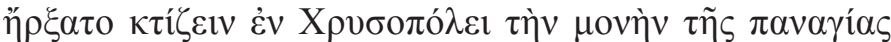

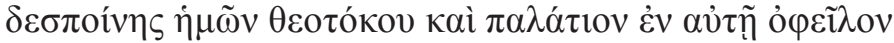

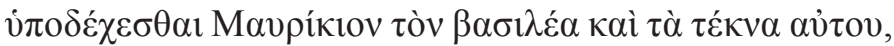

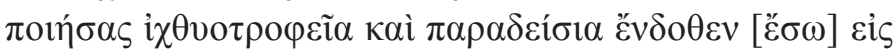

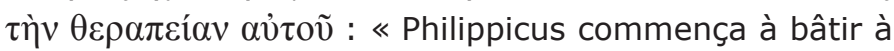
Chrysopolis le monastère de notre très sainte maîtresse la Mère de Dieu et un palais attenant pour la réception de l'empereur Maurice et ses enfants. Il y installa des viviers et des jardins pour son plaisir ».

JANin 1975, p. 24-25 ; HatLie 2007, p. 188-189.

\section{- Pierre le Patrice}

\section{PLRE IIIB : Petrus 6}

Maître des offices en 539-565, patrice en 542, consul honoraire en 552 .

\section{Constantinople}

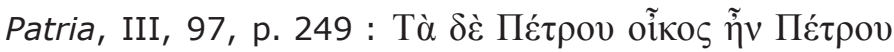

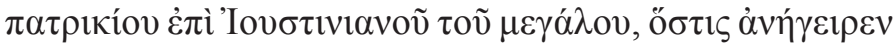

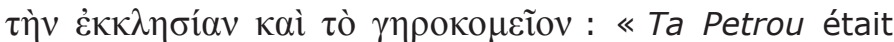


I'oikos du patrice Petrus au temps de Justinien le Grand, ce Petrus qui bâtit une église et un hospice pour vieillards ».

JANin 1964, p. 408 ; Janin 1969, p. 556 ; Berger 1988, p. 350-352 ( $\left.n^{\circ} 57\right)$.

L'oikos de Pierre le Patrice est situé entre le forum du Bœuf et le Philadelphion.

2.Akonai (île en face de Chalcédoine)

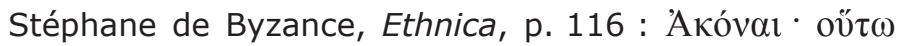

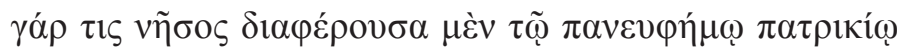

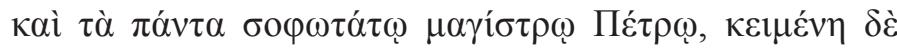

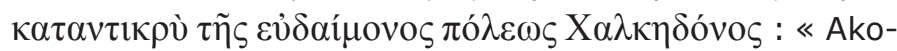
nai : une île appartenant ainsi en effet au très fameux patrice et très sage en tout, le magister Petrus, située en face de I'heureuse cité de Chalcédoine ».

\section{- Pierre Barsymès}

\section{PLRE IIIB : Petrus qui et Barsymes 9}

Consul honoraire et patrice en 542, préfet du prétoire d'Orient en 553-562.

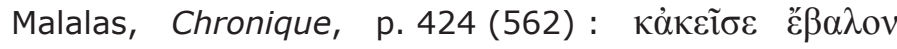

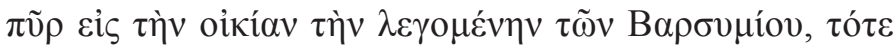

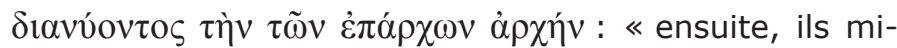
rent le feu à la maison dite de ta Barsymiou, [d'après celui] qui exerçait alors la préfecture ».

Il construit un palais à Constantinople (peut-être pour remplacer le précédent) qui fut ensuite donné par Maurice à sa sœur : Jean d'Éphèse, Histoire ecclésiastique, III, 5, 18 : «Tandis que son autre sœur, la veuve, reçut une maison nouvelle et solidement bâtie, celle du patrice Petrus, celui qu'on appelait Barsaumia, qu'il avait construite récemment et qui était comme une ville » (trad. C. Fauchon).

\section{- Platon}

PLRE II : PLATO 2

\section{Cubicularius sous Basiliscus}

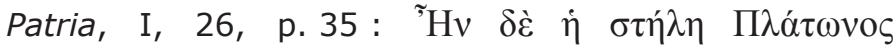

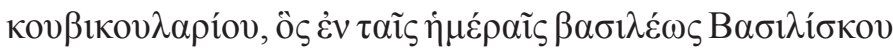

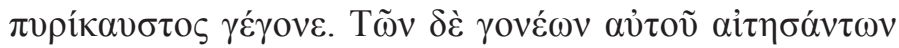

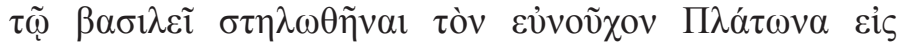

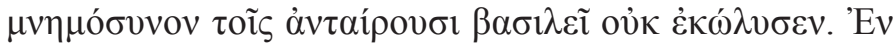

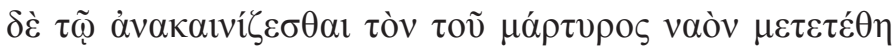

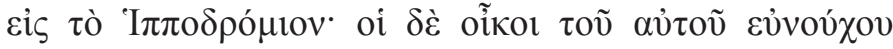

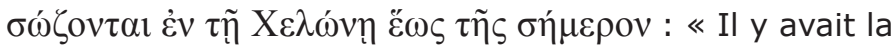
statue du cubicularius Platon, qui fut brûlée au temps de I'empereur Basiliscus. Comme ses parents demandaient à l'empereur que l'eunuque Platon soit célébré par une colonne en témoignage pour ceux qui se dressent contre l'empereur, ce dernier ne n'y opposa pas. Comme l'église du martyr était rénovée, elle fut déplacée à I’hippodrome. Les oikoi de cet eunuque existent encore de nos jours à Chélônè ».

Janin 1964, p. 414 ; Berger 1988, p. 460-462 ( $n^{\circ} 110$ ). L'oikos de Platon est situé à Chélônè près du Zeugma.

\section{- Pompeius}

\section{PLRE II : Pompeius 2}

Neveu d'Anastase.

Consul en 501, patrice.

Marcellinus comes, Chronique, a. 512 : sa maison (domus) est incendiée lors de l'émeute.

JANIN 1964, p. 414.

\section{- Priscus}

PLRE IIIB : Priscus 6

Époux de Domentzia, fille de Phocas.

Maître des milices en 588-599, comes excubitorum en 602-612.

Jean d'Antioche, fr. Roberto, p. 552, I. 21-23 (4 oc-

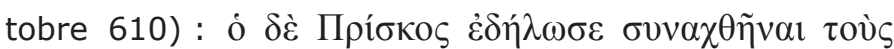

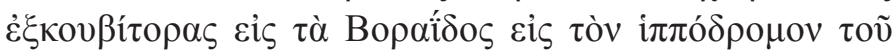

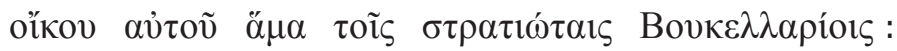
«Priscus commit le méfait de rassembler les excubitores avec les soldats bucellaires à ta Boraidos dans I'hippodrome de son oikos ».

JANin 1964, p. 325-326.

Ta Boraidou est situé au nord du port Sophien.

\section{- Probus}

\section{PLRE II : Fl. Probus 8}

Neveu d'Anastase.

Consul en 502, patrice.

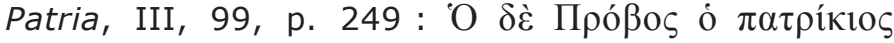

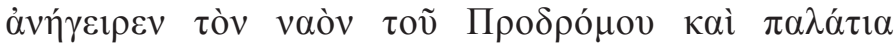

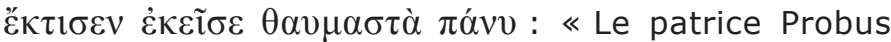
bâtit l'église du Prodrome et construisit là un palais tout à fait merveilleux ».

JANIN 1964, p. 416 ; JANIN 1969, p. 462 ; BERgER 1988, p. 745-746.

Les biens de Probus sont situés juste au nord de ta Boraidou et du port Sophien.

\section{- Rufinus}

\section{PLRE II : Rufinus 13}

Magister militum per Thracias en 515, patrice. Cyrille de Scythopolis, Vie de Sabas, p. 145, I. 6-7:

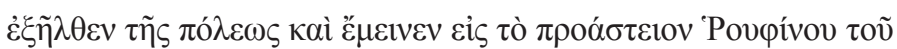
$\kappa \alpha \tau \alpha ̀ \Delta \eta \mu o ́ \sigma \tau \rho \alpha \tau o v$ : «Il sortit de Constantinople et séjourna dans le domaine de Rufinus, lié à Demostratus ». Demostratus était certainement le frère de Rufinus.

FEISSEL 2005, p. 256-260.

On ne sait où se trouvait le proasteion de Rufinus mais I'identification avec les Rufinianae doit être écartée. 
- Rufus

PLRE II : Fl. Rufus 4 et FI. Rufus 5

Consul en 457 ou en 492.

\section{Constantinople}

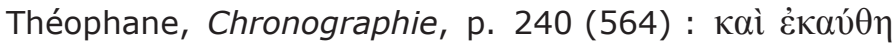

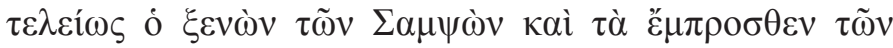

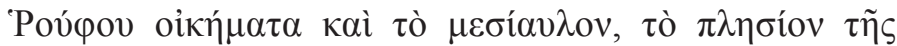

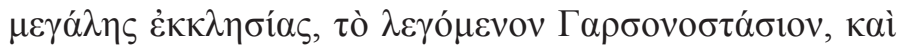

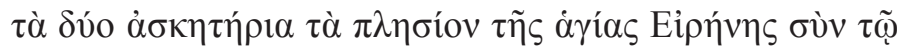

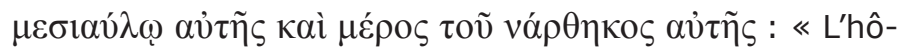
pital de Sampson fut complètement incendié, de même que les habitations situées sur le devant de ta Rouphou ainsi que la cour centrale proche de la grande église, celle qui est appelée Garsonostasion, et les deux monastères près de Sainte-Irène, de même que sa cour centrale et une partie de son narthex ».

JANIN 1964, p. 421.

Ta Rouphou est situé au nord-est de Sainte-Sophie.

\section{Chalcédoine}

PARgoire, 1898 ; Janin 1964, p. 504.

Ta Rouphou est situé aux environs de Chalcédoine, peutêtre à Bulgurlu.

\section{- Smaragdus}

\section{PLRE IIIB : Smaragdus 2}

Exarque d'Italie en 584/585-589/590 et 603-608, patrice.

Patria, III, 197, p. 277 : Tò $\delta \dot{\varepsilon} \Sigma \mu \alpha \rho \alpha ́ \gamma \delta \eta \varsigma \sigma u ̀ v ~ \tau \tilde{\omega} \lambda$ $\lambda o \varepsilon \tau \rho \tilde{\omega}$

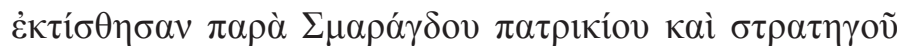

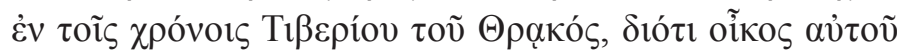
ทे $v \varepsilon \kappa \varepsilon i ̃ \sigma \varepsilon:$ :Ta Smaragdès fut construit avec un bain par le patrice Smaragdus, stratègos au temps de Tibère le Thrace, car son oikos se trouvait là ».

Le terme de stratègos est manifestement mis pour exarque.

JANin 1964, p. 222, 426 ; Berger 1988, p. 748-749.

L'oikos de Smaragdus est situé au nord-ouest de la citerne de Saint-Mokios.

\section{- Sophie}

\section{PLRE IIIB : Aelia Sophia 1}

Épouse de Justin II.

Corippe, Éloge de l'empereur Justin II, IV, 271 : Theodora regebat tempore quo sacram fundauit conditor arcem et Sophiae nomen nondum regnantis habere arx meruit : sceptri fuerant ea signa futuri : «Théodora gouvernait au temps où cette citadelle sacrée fut établie par son fondateur et où le nom de Sophie, qui ne régnait pas encore, lui revint : tel avait été le signe du sceptre à venir » (trad. S. Antès).

JANIN 1964， p. 134，153； BERgER 1988， p. 435$438\left(n^{\circ} 99\right)$.
Les Sophianai sont situés sur la Mésè près de ta Dareiou et de ta Basiliskou.

\section{- Sporacius}

\section{PLRE II : FI. Sporacius 3}

Comes domesticorum peditum en 450-451, consul en 452.

Patria, III, 30, p. 225-226 : Tòv $\delta \dot{\varepsilon}$ ă $\gamma 10 v \Theta \varepsilon o ́ \delta \omega \rho o v$

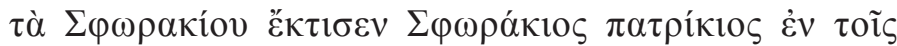

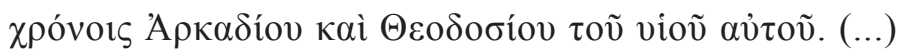

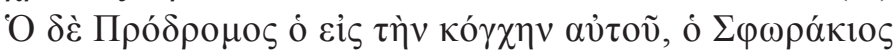

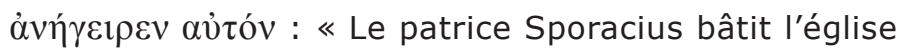
Saint-Théodore ta Sphôrakiou au temps d'Arcadius et de son fils Théodose (...) Quant au Prodrome dans I'abside, c'est Sporacius qui I'éleva ».

JANin 1964, p. 428 ; Janin 1969, p. 152 ; Berger 1988, p. $280-282$ ( $\left.n^{\circ} 25\right)$.

Les biens de Sporacius sont situés immédiatement à l'ouest du Milion.

\section{- Studius}

\section{PLRE II : Studius 2}

Consul en 454, patrice.

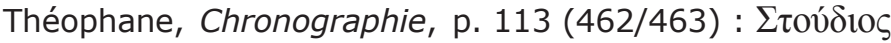

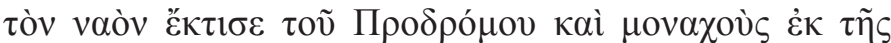

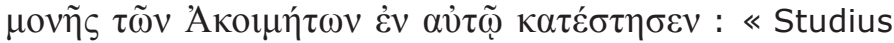
bâtit l'église du Prodrome et y établit des moines du monastère des Acémètes ».

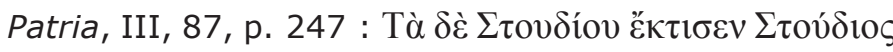

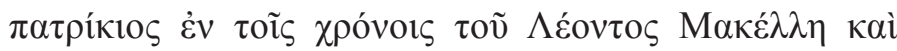

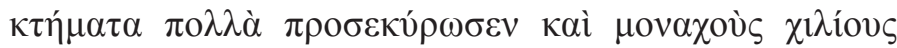
$\pi \varepsilon \pi$ oí $\kappa_{\varepsilon \nu}$ : «Le patrice Studius bâtit ta Stoudiou au temps de Léon le boucher, il accorda de nombreux biens et installa mille moines ».

Anthologie grecque, I, 4, p. 14 : «C'est Stoudios qui a élevé cette belle demeure (oĩkov) à Jean, le grand serviteur du Christ ; mais bientôt il a trouvé la récompense de ses travaux, en recevant les faisceaux consulaires » (trad. P. Waltz).

JAnin 1964, p. 431 ; Berger 1988, p. 363-365 ( $\left.n^{\circ} 64\right)$; Mango 1978 ; Hatlie 2007, p. 189.

Studius a bâti l'église, certainement sur ses biens, sans doute avant 454 et fut peut-être récompensé par le consulat. La construction se placerait donc sous Marcien et non sous Léon I ${ }^{\text {er }}$ comme le disent Théophane et, certainement à sa suite, les Patria.

\section{- Theoctista}

\section{PLRE IIIB : Theoctista 2}

Sœur de Maurice.

Jean d'Éphèse, Histoire ecclésiastique, III, 5, 18 : « Tandis que son autre sœur, la veuve, reçut une maison nouvelle 
et solidement bâtie, celle du patrice Petrus, celui qu'on appelait Barsaumia, qu'il avait construite récemment et qui était comme une ville » (trad. C. Fauchon).

- Urbicius (praepositus)

\section{PLRE II : Vrbicius 1}

Praepositus sacri cubiculi en 449, vers 470-481, en 491 ; ex consul.

AvRAMÉA 1987, p. 399-401: plaque de marbre découverte dans la banlieue européenne de Constantinople,

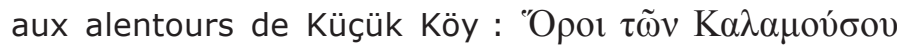

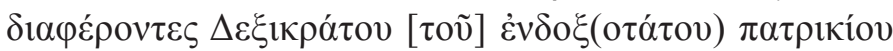

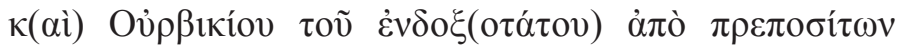
$[\hat{\alpha}] \pi$ ò íá $\tau \omega v$ : «Bornes de ta Kalamousou appartenant à Dexicrates, le très glorieux patrice, et à Ourbikios, le très glorieux ex-préposite, ex-consul ».

Nous ignorons quel est le domaine appelé ta Kalamousou.

- Urbicius (maître des milices)

PLRE II : Vrbicius qui et Barbatus 2

Patrice, consul honoraire et magister militum per Orientem sous Anastase.

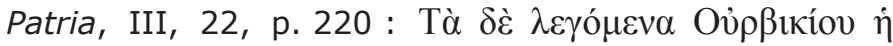

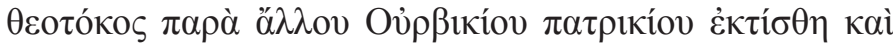

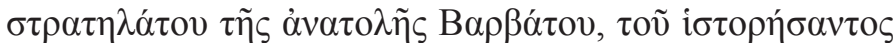

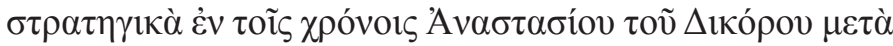

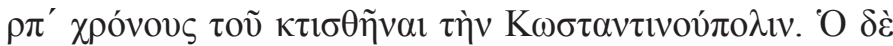

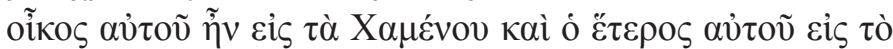
$\Sigma \tau \rho \alpha \tau \eta ́ \gamma 10 v$ : «Ce que l'on appelle ta Ourbikiou [à savoir] la Théotokos a été construite par un autre patrice Urbicius, homme barbu [non eunuque], stratèlatès d'Orient et qui écrivit des traités stratégiques au temps d'Anastase le Dikoros 180 ans après la fondation de Constantinople. L'un de ses oikoi se trouvait à ta Chaménou et I'autre au Stratègion ».

JANIN 1964, p. 329, 400 ; JANIN 1969, p. 216-217 ; BERGER 1988, p. 404-406 ( $\left.n^{\circ} 86\right)$.

L'un des oikoi du maître des milices Urbicius est situé au Stratègion ; la localisation de l'autre à ta Chaménou est inconnue.

\section{- Varanes}

PLRE II : Varanes 1 ou Varanes (Baranes) 2

Consul en 410 ou 456.

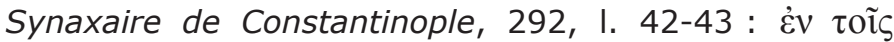

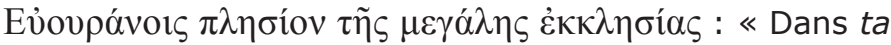
Euouranois près de la grande église ».

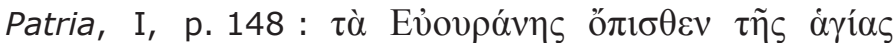
бopías : «ta Euouranès derrière Sainte-Sophie ».

JANIN 1964, p. 400 ; JANIN 1969, p. 184-185.

Les biens de Varanes sont situés près de Sainte-Sophie, derrière la basilique.

\section{- Vigilantia}

\section{PLRE II : Vigilantia}

Sœur de Justinien, mère de Justin II.

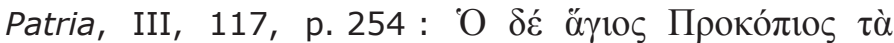

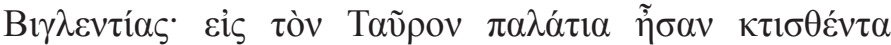

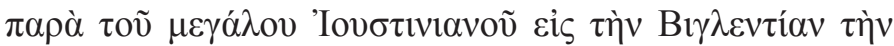

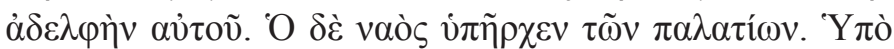

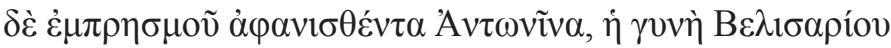

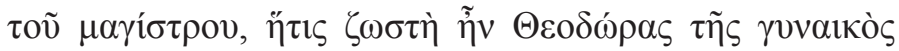

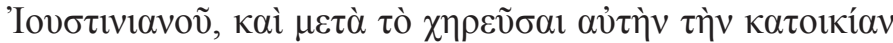

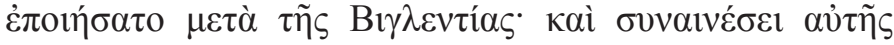

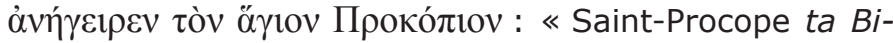
glentias : au [forum] Tauri se trouvait un palais bâti par Justinien le Grand pour sa sœur Vigilantia. L'église appartenait au palais. Comme elle avait été détruite par un incendie et alors qu'Antonina, femme du magister Bélisaire et zôstè de Théodora épouse de Justinien, cohabitait après son veuvage avec Vigilantia, elle bâtit SaintProcope avec l'accord de celle-ci ».

L'anecdote concernant Antonina n'est pas sûre d'autant plus que le terme de zôstè («à ceinture ») pour qualifier une patricienne est anachronique.

JANin 1964, p. 323 ; Berger 1988, p. 504-506 ( $n^{\circ} 131$ ).

Ta Biglentias se trouve au nord du forum de Théodose et à l'ouest du portique de Domninos. 


\section{BIBLIOGRAPHIE}

\section{SOURCES}

ACO, IV : Acta Conciliorum Ecumenicorum, IV (Constantinople II), éd. J. Straub, Berlin, 1970-1974.

Agathias, Histoires : Agathiae Myrinaei Historiarum libri quinque, éd. R. Keydell, Berlin, 1967.

Anthologie grecque, tome I, Anthologie palatine, livres I-IV, éd. et trad. P. Waltz, Paris, 1929.

Anthologie grecque, tome VIII, Anthologie palatine, livre IX, épigr. 359-827, éd et trad P. Waltz \& G. Soury, Paris, 1974.

Avraméa, Anna, 1987, « Bornes de propriétés foncières de Constantinople et de Thrace », Travaux et Mémoires 10, p. 399404.

Chronique Pascale : Chronicon paschale, éd. L. Dindorf, Bonn, 1832.

Corippe, Éloge de l'empereur Justin II, éd. et trad. S. Antès, Paris, 1981.

Cyrille de Scythopolis, Vie de Sabas : SchwARTz, Eduard, 1939, Kyrillos von Skythopolis, Leipzig.

Festugière, André-Jean, 1961, Les moines d'Orient, II, Paris.

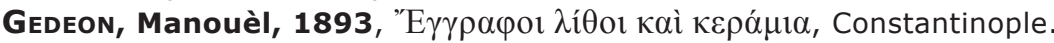

Jean d'Antioche, fr. Roberto : Ioannis Antiocheni Fragmenta ex Historia chronica, éd. et trad. U. Roberto, Berlin - New York, 2005.

Jean d'Antioche, fr. Mariev : Ioannis Antiocheni Fragmenta quae supersunt omnia, éd. et trad. S. Mariev, Berlin - New York, 2008.

Jean d'Éphèse, Histoire ecclésiastique : Iohannis Ephesini Historiae ecclesiasticae pars tertia, éd. E. W. Brooks, Paris, 1936. Malalas, Chronique : Ioannis Malalae Chronographia, éd. I. Thurn, Berlin - New York, 2000.

Marcellinus comes, Chronique : Marcellini comitis chronicon, éd. Th. Mommsen, dans Monumenta Germaniae Historica, Auctores Antiquissimi, XI, Berlin, 1894, p. 60-108.

Maraval, Pierre, 2007, Agathias, Histoires, Guerres et malheurs du temps sous Justinien, Paris.

Novelles : Corpus Iuris Civilis, III, Novellae, éd. R. Schoell \& W. Kroll, Berlin, 1895.

Olympiodore, fr. : The Fragmentary Classicizing Historians of the Later Roman Empire, II, éd. et trad. R. C. Blockley, Cambridge, 1983.

Patria : Scriptores originum Constantinopolitanarum, éd. Th. Preger, Leipzig, 1901-1907.

Procope, Édifices : Procopius, Buildings, éd. J. Haury, trad. H. B. Dewing, Cambridge (Mass.) - London, 1940.

Procope, Guerre contre les Perses : Procopius, History of the Wars. Books I-II, éd. J. Haury, trad. H. B. Dewing, Cambridge (Mass.) - London, 1914.

Procope, Guerre contre les Vandales: Procopius, History of the Wars. Books III-IV, éd. J. Haury, trad. H. B. Dewing, Cambridge (Mass.) - London, 1916.

Procope, Guerre contre les Goths : Procopius, History of the Wars. Books V-VIII, éd. J. Haury, trad. H. B. Dewing, Cambridge (Mass.) - London, 1919-1928.

Procope, Histoire secrète : Procopius, Secret History, éd. J. Haury, trad. H. B. Dewing, Cambridge (Mass.) - London, 1935.

Roques, Denis, 2011, Procope de Césarée, Constructions de Justinien I ${ }^{e r}$, Alessandria.

Stéphane de Byzance, Ethnica : Stephani Byzantii Ethnica, volumen I : A- $\Gamma$, éd. et trad. M. Billerbeck, Berlin - New York, 2006.

Synaxaire de Constantinople : Synaxarium Ecclesiae Constantinopolitanae, éd. H. Delehaye, Bruxelles, 1900.

Théophane, Chronographie: Theophanis chronographia, éd. C. de Boor, Leipzig, 1883-1885.

Vie de Daniel le Scétiote : Vie (et récits) de l'abbé Daniel le Scétiote (VIe siècle), éd. L. Clugnet, Paris, 1901.

Vie de Daniel le Stylite : Delehaye Hippolyte, 1923, Les saints stylites, Bruxelles, p. 1-94. 
Vie d'Olympias: Jean Chrysostome, Lettres à Olympias, Vie anonyme d'Olympias, éd. et trad. A.-M. Malingrey, Paris, 1968.

Vie de Théodore de Sykéôn, éd. et trad. A.-J. Festugière, Buxelles, 1970.

Zonaras, Histoires : Ioannes Zonaras, Epitome Historiarum, éd. L. Dindorf, Leipzig, 1868-1875.

\section{ÉTUDES}

Berger, Albrecht, 1988, Untersuchungen zu den Patria Konstantinupoleos, Bonn.

CABouret, Bernadette, 2013, «Élites de I'Orient romain à la campagne dans I'Antiquité tardive », dans Gérard Charpentier \& Vincent Puech (éd.), Villes et campagnes aux rives de la Méditerranée ancienne. Hommages à Georges Tate, Topoi, Suppl. 12, p. 421-457.

CarriÉ, Jean-Michel, 1999, «Économie et société de l'Égypte romano-byzantine (IV -VII ${ }^{e}$ siècle). À propos de quelques publications récentes », Antiquité Tardive 7, p. 331-352.

Čekalova, Alexandra, 1998, « Fortune des sénateurs de Constantinople du IV e au début du VII siècle », dans EYYYXIA. Mélanges offerts à Hélène Ahrweiler, I, Paris, p. 119-130.

Cheynet, Jean-Claude, 1991, « Fortune et puissance de l'aristocratie ( $x^{\mathrm{e}}-\mathrm{XII}{ }^{\mathrm{e}}$ siècle) 》, dans Vassiliki Kravari, Jacques Lefort \& Cécile Morrisson (éd.), Hommes et richesses dans l'Empire byzantin II, Paris, p. 199-214 (repris dans The Byzantine Aristocracy and its Military Function, Aldershot, 2006).

Cheynet, Jean-Claude, 2003, « L'époque byzantine », dans Bernard Geyer \& Jacques Lefort (éd.), La Bithynie au Moyen Âge, Paris, p. 311-350.

DAGRON, Gilbert, 1974, Naissance d'une capitale. Constantinople et ses institutions de 330 à 451, Paris.

Dagron, Gilbert, 1977, «Le christianisme dans la ville byzantine », Dumbarton Oaks Papers 31, p. 3-25 (repris dans La romanité chrétienne en Orient : héritages et mutations, Londres, 1984).

DAGRon, Gilbert, 1984, Constantinople imaginaire. Études sur le recueil des « Patria », Paris.

DelmaIre, Roland, 1989a, Largesses sacrées et res privata. L'aerarium impérial et son administration du IVe au vi siècle (Collection de I'École française de Rome 121), Rome.

DelmaIRE, Roland, 1989b, Les responsables des finances impériales au Bas-Empire romain (IVe-VIe s.). Études prosopographiques (Collection Latomus 203), Bruxelles.

Destephen, Sylvain, 2012, «L'évergétisme aristocratique au féminin dans l'Empire romain d'Orient », dans Béatrice Caseau (éd.), Les réseaux familiaux, Antiquité tardive et Moyen Âge. In memoriam A. Laiou et É. Patlagean, Paris, p. 183-203.

Donciu, Ramiro, 2012, L'empereur Maxence, Bari.

Duвouloz, Julien, 2011, La propriété immobilière à Rome et en Italie ( $I^{\text {er }}-v^{e}$ siècles). Organisation et transmission des praedia urbana (Bibliothèque des Écoles françaises d'Athènes et de Rome 343), Rome.

ÉtIENNE, Roland, 2013, «La notion de proasteion dans les textes grecs », dans Pascal Darcque, Roland Étienne \& Anne-Marie Guimier-Sorbets (éd.), PROASTEION. Recherches sur le périurbain dans le monde grec, Paris, p. 13-27.

Ferssel, Denis, 1999, «Deux grandes familles isauriennes du ve siècle d'après des inscriptions de Cilicie Trachée », Mitteilungen zur Christlichen Archäologie 5, p. 9-17.

Feissel, Denis, 2005, «De Sainte-Irène au domaine de Rufin. Trois notes de toponymie constantinopolitaine », Travaux et Mémoires 15, p. 245-260.

Flusin, Bernard, 1998, «Évêques et patriarches - Les structures de l'Église impériale » dans Luce Pietri (éd.), Histoire du christianisme, Tome III. Les Églises d'Orient d'Occident (432-610), Paris, p. 485-543.

Gascou, Jean, 1985, «Les grands domaines, la cité et l'État en Égypte byzantine », Travaux et Mémoires 9, p. 1-90 (repris dans Fiscalité et société en Égypte byzantine, Paris 2008, p. 125-213).

Gros, Pierre, 2006, L'architecture romaine du début du III siècle av J.-C. à la fin du Haut-Empire. 2. Maisons, palais, villas et tombeaux, $2^{\mathrm{e}}$ éd. (1 1 ère éd. 2001), Paris.

Hatlie, Peter, 2007, The Monks and Monasteries of Constantinople, ca. 350-850, Cambridge.

HickeY, Todd, 2007, « Aristocratic landholding and the economy of Byzantine Egypt », dans Roger Bagnall (éd.), Egypt in the Byzantine World, 300-700, Cambridge, p. 288-308.

Janin, Raymond, 1964, Constantinople byzantine. Développement urbain et répertoire topographique, $2^{\mathrm{e}}$ éd. (1 $1^{\mathrm{è} e}$ éd. 1950 ) (Archives de I'Orient chrétien 4A), Paris.

JANIN, Raymond, 1969, La géographie ecclésiastique de l'Empire byzantin. Première partie : Le siège de Constantinople et le patriarcat œcuménique. Tome III : Les églises et les monastères, $2^{\mathrm{e}}$ éd. (1 1 èr éd. 1953), Paris.

Janin, Raymond, 1975, Les églises et les monastères des grands centres byzantins (Bithynie, Hellespont, Latros, Galèsios, Trébizonde, Athènes, Thessalonique) (Géographie ecclésiastique de l'Empire byzantin 2), Paris.

KaPLAN, Michel, 1986, «Quelques aspects des maisons divines du VI ${ }^{\mathrm{e}}$ au IX ${ }^{\mathrm{e}}$ siècle », Mélanges Svoronos, Rethymnon, p. 7096 (repris dans Byzance. Villes et campagnes, Paris 2006, p. 138-156).

KAPLAN, Michel, 1992, Les hommes et la terre à Byzance du VI au XI siècle. Propriété et exploitation du sol (Publications de la Sorbonne, Série Byzantina Sorbonensia 10), Paris.

Magdalino, Paul, 1974, «The Byzantine Aristocratic Oikos », dans Michael Angold (éd.), The Byzantine Aristocracy: IX-xIII Centuries, Oxford, p. 92-111.

Magdalino, Paul, 1996, Constantinople médiévale. Étude sur l'évolution des structures urbaines, Paris.

Mango, Cyril, 1978, «The Date of the Studius Basilica at Istanbul », Byzantine and Modern Greek Studies 4, p. $115-122$.

Morrisson, Cécile (dir.), 2004, Le monde byzantin I. L'Empire romain d'Orient (330-341), Paris. 
Morvillez, Éric, 2014, «Les transformations du jardin de tradition romaine dans l'Antiquité tardive », dans Paul Van Ossel \& Anne-Marie Guimier-Sorbets (éd.), Archéologie des jardins. Analyse des espaces et méthodes d'approche, Montagnac, p. 161176.

Pargoire, Jules, 1898, «Environs de Chalcédoine », Échos d'Orient I, p. 145-147.

PLRE I : Jones, Arnold, Martindale John \& MorRis, John, 1971, The Prosopography of the Later Roman Empire. Volume I, A. D. 260-395, Cambridge.

PLRE II : MARTINDALE, John, 1980, The Prosopography of the Later Roman Empire. Volume II, A. D. 395-527, Cambridge. PLRE IIIA et PLRE IIIB : MARTINDALe, John, 1992, The Prosopography of the Later Roman Empire. Volume III, A.D. 527641, Cambridge.

Saliou, Catherine, 2000, « Mesurer le paradis. Contribution au portrait d'Antioche aux époques romaine et protobyzantine », dans Claude Nicolet (éd.), Mégapoles méditerranéennes, Paris, p. 802-819.

Saliou, Catherine, 2014, «Aux limites du jardin. Le droit et les limites du jardin dans le monde romain », dans Paul Van Ossel \& Anne-Marie Guimier-Sorbets (éd.), Archéologie des jardins. Analyse des espaces et méthodes d'approche, Montagnac, p. 195202. 